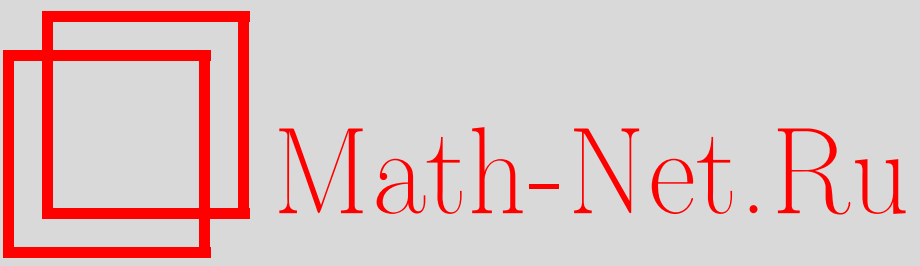

В. А. Ватутин, К. Лиу, Критические ветвящиеся процессы с двумя типами частиц, эволюционирующие в асинхронных случайных средах, Теория вероятн. и ее примен., 2012, том 57, выпуск 2, 225-256

DOI: https://doi.org/10.4213/tvp4445

Использование Общероссийского математического портала Math-Net.Ru подразумевает, что вы прочитали и согласны с пользовательским соглашением

http: //www . mathnet.ru/rus/agreement

Параметры загрузки:

IP : 3.81 .55 .215

26 апреля 2023 г., 14:52:10 
ТЕОРИЯ ВЕРОЯТНОСТЕЙ

Том 57

И ЕЕ ПРИМЕНЕНИЯ

Выпуск 2

2012

(c) 2012 г.

ВАТУТИН В. А.* , ЛИУ К.**

\title{
КРИТИЧЕСКИЕ ВЕТВЯЩИЕСЯ ПРОЦЕССЫ С ДВУМЯ ТИПАМИ ЧАСТИЦ, ЭВОЛЮЦИОНИРУЮЩИЕ В АСИНХРОННЫХ СЛУЧАЙНЫХ СРЕДАХ ${ }^{1)}$
}

\begin{abstract}
Рассматривается полностью разложимый ветвящийся процесс в случайной среде с двумя типами частиц. Частицы в этом процессе могут порождать потомков лишь своего собственного типа. Обозначим $\exp \left\{X_{k}(i)\right\}$ среднее число потомков, порождаемых одной частицей типа $i=1,2$ поколения $k$. Предполагая, что $X_{k}(2)=-X_{k}(1)$ с вероятностью единица, и что случайное блуждание $S_{n}(1)=$ $X_{1}(1)+\cdots+X_{n}(1)$, порождаемое случайной средой, является осциллирующим, мы исследуем совместное условное распределение числа частиц обоих типов в популяции в моменты $n t, 0<t \leqslant 1$, при условии, что оба типа не выродились к моменту $n \rightarrow \infty$. При тех же условиях мы находим асимптотическое представление для совместных условных распределений размеров популяций обеих типов в моменты, когда состояние среды является чрезвычайно неблагоприятными для частиц первого типа. Показано, что рассматриваемый процесс обладает необычными свойствами, которые могут трактоваться как бутылочные горлышки и периоды роста в модели сосуществования хищника и жертвы.
\end{abstract}

Ключевые слова и фразы: ветвящиеся процессы в случайной среде, условные предельные теоремы, бутылочные горлышки.

1. Введение. Модель ветвящихся процессов в случайной среде, рассматриваемая в данной работе, является аналогом (для случая процессов с двумя типами частиц) ветвящихся процессов, введенных Смитом и Вилкинсоном [7].

Для формального описания этой модели обозначим $\mathscr{M}$ пространство вероятностных мер на множестве $\mathbb{N}_{0}:=\{0,1,2, \ldots\}$ и пусть $\mathbf{Q}:=$ $\left(Q^{(1)}, Q^{(2)}\right)$ - вектор, компоненты которого принимают значения в пространстве $\mathscr{M}$. Будем говорить, что бесконечная последовательность $\boldsymbol{\Pi}=\left(\mathbf{Q}_{\mathbf{1}}, \mathbf{Q}_{\mathbf{2}}, \ldots\right)$ независимых и одинаково распределенных копий $\mathbf{Q}$

\footnotetext{
* Математический институт им. В. А. Стеклова, ул. Губкина 8, 119991 Москва, Россия; e-mail: vatutin@mi.ras.ru

** Université de Bretagne-Sud, Laboratoire de Mathématiques de Bretagne Atlantique (LMBA), UMR 6205 Campus de Tohannic, BP 573, 56017, Vannes, France; e-mail: Quansheng.Liu@univ-ubs.fr

1) Работа выполнена при частичной поддержке РФФИ, грант 11-01-00139.
} 
образует случайную среду. Последовательность $\mathbb{N}_{0} \times \mathbb{N}_{0}$-значных случайных векторов $\mathbf{Z}_{0}, \mathbf{Z}_{1}, \ldots$ называется полностью разложимыл ветвящимся прочессом в случайной среде П с двумя типами частиц, если начальное значение $\mathbf{Z}_{0}=\left(Z_{0}^{(1)}, Z_{0}^{(2)}\right)$ не зависит $\boldsymbol{\Pi}$ и, при заданной среде $\boldsymbol{\Pi}$, процесс $\mathscr{Z}=\left(\mathbf{Z}_{0}, \mathbf{Z}_{1}, \ldots\right)$ с компонентами $\mathbf{Z}_{n}=\left(Z_{n}^{(1)}, Z_{n}^{(2)}\right), n=0,1, \ldots$, является цепью Маркова, задаваемой равенством

$$
\begin{array}{r}
\mathscr{L}\left(\mathbf{Z}_{n} \mid \mathbf{Z}_{n-1}=\left(z_{n-1}^{(1)}, z_{n-1}^{(2)}\right), \mathbf{\Pi}=\left(\mathbf{Q}_{\mathbf{1}}, \mathbf{Q}_{\mathbf{2}}, \ldots\right)\right) \\
=\mathscr{L}\left(\xi_{n 1}^{(1)}+\cdots+\xi_{n z_{n-1}^{(1)}}^{(1)}, \xi_{n 1}^{(2)}+\cdots+\xi_{n z_{n-1}^{(2)}}^{(2)}\right)
\end{array}
$$

для любых $n \geqslant 1,\left(z_{n-1}^{(1)}, z_{n-1}^{(2)}\right) \in \mathbb{N}_{0} \times \mathbb{N}_{0}$ и $\mathbf{Q}_{n}=\left(q_{n}^{(1)}, q_{n}^{(2)}\right) \in \mathscr{M} \times \mathscr{M}$, где $\xi_{n 1}^{(i)}, \xi_{n 2}^{(i)}, \ldots$ - независимые одинаково распределенные случайные величины с распределением $q_{n}^{(i)}, i=1,2$. Мы можем записать этот факт в виде

$$
Z_{n}^{(i)}:=\sum_{i=1}^{Z_{n-1}^{(i)}} \xi_{n i}^{(i)}, \quad i=1,2,
$$

где, при фиксации среды, $Z_{n}^{(1)}$ и $Z_{n}^{(2)}$ являются обычными неоднородными ветвящимися процессами Гальтона-Ватсона. Таким образом, случайная величина $\mathbf{Z}_{n}$ - это размер $n$-го поколения популяции, а $\mathbf{Q}_{n}-$ вектор, задающий распределение числа потомков индивидуумов соответствующих типов в поколении $n-1$. Заметим, что частицы интересующего нас процесса производят потомков только своего типа. Обозначим $\mathbf{P}$ соответствующую вероятностную меру на рассматриваемом вероятностном пространстве.

В дальнейшем мы отождествляем наборы $\mathbf{Q}$ и $\mathbf{Q}_{n}, n=1,2, \ldots$, с парами (случайных) производящих функций

$$
f^{(i)}(s):=\sum_{k=0}^{\infty} s^{k} Q^{(i)}(\{k\})=: \mathbf{E}\left[s^{\xi^{(i)}} \mid Q^{(i)}\right], \quad 0 \leqslant s \leqslant 1, \quad i=1,2,
$$

и

$$
f_{n}^{(i)}(s):=\sum_{k=0}^{\infty} s^{k} Q_{n}^{(i)}(\{k\})=: \mathbf{E}\left[s^{\xi_{n}^{(i)}} \mid Q_{n}^{(i)}\right], \quad 0 \leqslant s \leqslant 1, \quad i=1,2,
$$

соответственно, и не делаем различия между наборами $\boldsymbol{\Pi}=\left(\mathbf{Q}_{\mathbf{1}}, \mathbf{Q}_{\mathbf{2}}, \ldots\right)$ и $\left(\mathbf{f}^{(1)}, \mathbf{f}^{(2)}\right):=\left\{\left(f_{n}^{(1)}, f_{n}^{(2)}\right), n=1,2, \ldots\right\}$.

Положим

$$
X(i):=\ln \mathbf{E}\left[\xi^{(i)} \mid Q^{(i)}\right], \quad i=1,2,
$$

и

$$
X_{k}(i):=\ln \mathbf{E}\left[\xi^{(i)} \mid Q_{k}^{(i)}\right], \quad \eta_{k}(i):=\frac{\mathbf{E}\left[\xi_{k}^{(i)}\left(\xi_{k}^{(i)}-1\right) \mid Q_{k}^{(i)}\right]}{2\left(\mathbf{E}\left[\xi_{k}^{(i)} \mid Q_{k}^{(i)}\right]\right)^{2}}, \quad k=1,2, \ldots
$$


Одним из наших основных предположений является следующее условие.

У с л о в и е $\mathrm{A} 1$. Для любого $k=1,2, \ldots$

$$
X_{k}(2)=-X_{k}(1) \quad \mathbf{P} \text {-a.s. }
$$

Таким образом, важным свойством рассматриваемой модели является тот факт, что процессы $Z^{(i)}(n), i=1,2$ зависимы только через среду и, согласно условию А1, эволюционируют асинхронно: благоприятная среда для первого процесса $\left(X_{k}(1)>0\right)$ является неблагоприятной для второго $\left(X_{k}(2)<0\right)$ и наоборот.

Введем два случайных блуждания

$$
S_{0}(i):=0, \quad S_{n}(i):=\sum_{k=1}^{n} X_{k}(i), \quad i=1,2 .
$$

Ясно, что $S_{n}(2)=-S_{n}(1), n=0,1, \ldots$

Для простоты мы используем в дальнейшем обозначения $S_{n}:=$ $S_{n}(1)$ и $X=X_{1}(1)$. Несложно переформулировать все утверждения, связанные со случайной величиной $S_{n}(1)$, в соответствующие утверждения для случайной величины $S_{n}(2)$.

У с л о в и е А2. Распределение случайной величины $X$ нерешетчато, и найдутся числа $c_{n} \rightarrow \infty$ такие, что последовательность $S_{n} / c_{n}$ сходится по распределению к устойчивой с параметром $\alpha$ случайной величине $Y$, причем $\alpha \in(0,1) \cup(1,2]$ и параметр положительности $\rho:=\mathbf{P}(Y>0) \in(0,1)$ таковы, что либо $\alpha \rho<1$, либо $\mathbf{E} X^{2}<\infty$.

Известно (см., например, [5, гл. XVII, §5]), что при выполнении условия А2 последовательность $\left\{c_{n}, n \geqslant 1\right\}$ правильно меняется с параметром $\alpha^{-1}$, т.е., найдется медленно меняющаяся на бесконечности функция $l(n)$ такая, что

$$
c_{n}=n^{1 / \alpha} l(n) .
$$

Более того, последовательность $\left\{c_{n}, n \geqslant 1\right\}$ может быть выбрана монотонно возрастающей и так, что при $n \rightarrow \infty$

$$
\mathbf{P}\left(X>c_{n}\right) \sim n^{-1} .
$$

Кроме того, если $\alpha<2$, то

$$
\mathbf{P}(X>x) \sim x^{-\alpha} L(x),
$$

где $L(x)$ - медленно меняющаяся на бесконечности функция, удовлетворяющая соотношению $L\left(x^{1 / \alpha} l(x)\right) \sim(l(x))^{\alpha}$ при $x \rightarrow \infty$.

Условие А2 означает, в частности, что $\left\{S_{n}, n \geqslant 0\right\}-$ осциллирующее случайное блуждание и, следовательно, ветвящиеся процессы $Z^{(i)}(n), i=1,2$ являются критическими согласно классификации, введенной в работе [1]. 
Введем случайные величины

$$
M_{n}:=\max \left(S_{1}, \ldots, S_{n}\right), \quad L_{n}:=\min \left(S_{1}, \ldots, S_{n}\right)
$$

и положим

$$
\begin{aligned}
& \mu(n):=\max \left\{1 \leqslant i \leqslant n: S_{i}=M_{n}\right\} \\
& \tau(n):=\min \left\{0 \leqslant i \leqslant n: S_{i}=\min \left(0, L_{n}\right)\right\} .
\end{aligned}
$$

Известно (см., например, [8, разд. 20]), что если условие А2 выполнено, то для любого $t \in(0,1]$ при $n \rightarrow \infty$

$$
\frac{\tau(n t)}{n} \stackrel{d}{\rightarrow} \tau_{t}, \quad \frac{\mu(n t)}{n} \stackrel{d}{\rightarrow} \mu_{t},
$$

где случайные величины $t^{-1} \tau_{t}$ и $t^{-1} \mu_{t}$ имеют обобщенные распределения арксинуса на интервале $[0,1]$ с плотностями

$$
\frac{1}{\Gamma(\rho) \Gamma(1-\rho)} \frac{1}{x^{\rho}(1-x)^{1-\rho}} \quad \text { и } \quad \frac{1}{\Gamma(\rho) \Gamma(1-\rho)} \frac{1}{x^{1-\rho}(1-x)^{\rho}} .
$$

Положим $D:=\sum_{j=1}^{\infty} j^{-1} \mathbf{P}\left(S_{j}=0\right)$ и обозначим $I\{A\}$ индикатор события $A$.

Пусть $\gamma_{0}:=0, \quad \gamma_{j+1}:=\min \left(n>\gamma_{j}: S_{n}<S_{\gamma_{j}}\right)$ и $\Gamma_{0}:=0, \quad \Gamma_{j+1}:=$ $\min \left(n>\Gamma_{j}: S_{n}>S_{\Gamma_{j}}\right), j \geqslant 0$, обозначают строгие нижние и верхние лестничные моменты случайного блуждания $\left\{S_{n}, n \geqslant 0\right\}$. Полагая $S_{0}=$ 0 , зададим функции $U: \mathbb{R} \rightarrow \mathbb{R}_{+}$и $V: \mathbb{R} \rightarrow \mathbb{R}_{+}$равенствами

$$
\begin{aligned}
& U(x):=I\{0<x\}+\sum_{j=1}^{\infty} \mathbf{P}\left(S_{\Gamma_{j}}<x\right), \quad x \in \mathbb{R}_{+}:=[0, \infty), \\
& U(x)=0, \quad x<0, \\
& V(x):=\sum_{j=0}^{\infty} \mathbf{P}\left(S_{\gamma_{j}} \geqslant-x\right), \quad x \in \mathbb{R}_{+}, \quad V(x)=0, \quad x<0 .
\end{aligned}
$$

Можно проверить, что $\lim _{x \rightarrow 0+} U(x)=U(0+)=e^{-D}$ и $\lim _{x \rightarrow+0} V(x)=V(0)=1$ (см., [10], где содержится доказательство этого утверждения).

Положим

$$
\Theta^{(i)}(r):=\frac{\sum_{y=r}^{\infty} y^{2} Q^{(i)}(\{y\})}{\left(\sum_{y=1}^{\infty} y Q^{(i)}(\{y\})\right)^{2}}, \quad r \in \mathbb{N}_{0}, \quad i=1,2 .
$$

У с ло в и е А3. Для $\varepsilon_{0}>0, r \in \mathbb{N}_{0}$ и $i=1,2$

$$
\mathbf{E}\left[\left(\ln ^{+} \Theta(r)\right)^{1 / \rho+\varepsilon_{0}}\right]+\mathbf{E}\left[\left(\ln ^{+} \Theta(r)\right)^{1 /(1-\rho)+\varepsilon_{0}}\right]<\infty
$$


И

$$
\mathbf{E}\left[U(-X)\left(\ln ^{+} \Theta(r)\right)^{1+\varepsilon_{0}}\right]+\mathbf{E}\left[V(X)\left(\ln ^{+} \gamma^{(i)}(r)\right)^{1+\varepsilon_{0}}\right]<\infty
$$

где $\ln ^{+} x:=\ln (\max \{x, 1\})$.

Обозначим $\mathbf{L}=\{\mathscr{L}\}$ множество всех (может быть, несобственных) вероятностных законов $\mathscr{L}=\mathscr{L}(\bar{\xi})$ случайных $b$-мерных векторов $\bar{\xi}=$ $\left(\xi_{1}, \ldots, \xi_{b}\right)$ с неотрицательными компонентами, и пусть $\mathbf{L}^{+} \subset \mathbf{L}-$ множество вероятностных законов из $\mathbf{L}$, соответствующих случайным векторам сосредоточенным на множестве $(0, \infty)^{b}: \mathbf{P}\left(\xi_{i}>0, i=1, \ldots, b\right)=1$. Пусть, далее, $\boldsymbol{\Phi}=\{\boldsymbol{\Phi}\}$ и $\boldsymbol{\Phi}^{+} \subset \boldsymbol{\Phi}-$ пространства преобразований Лапласа $\Phi(\bar{\lambda})=\int e^{-(\bar{\lambda}, \mathbf{x})} \mathscr{L}(d \mathbf{x}), \bar{\lambda}, \mathbf{x} \in[0, \infty)^{b}$, законов распределения из $\mathbf{L}$ и $\mathbf{L}^{+}$, соответственно, оснащенные метрикой $d_{\Lambda}, \Lambda>1$ :

$$
d_{\Lambda}\left(\Phi^{*}, \Phi^{* *}\right):=\sup _{\overline{1} \leqslant \bar{\lambda} \leqslant \bar{\Lambda}}\left|\Phi^{*}(\bar{\lambda})-\Phi^{* *}(\bar{\lambda})\right|
$$

где $\mathbf{1}=(1, \ldots, 1), \boldsymbol{\Lambda}=(\boldsymbol{\Lambda}, \ldots, \boldsymbol{\Lambda})-b$-мерные векторы, а неравенства между векторами понимаются покомпонентно. Поскольку преобразование Лапласа закона распределения случайного вектора с неотрицательными компонентами однозначно определяется своими значениями в любом $b$-мерном кубе, содержащемся в $(0, \infty)^{b}$, то сходимость $\Phi_{n} \rightarrow \Phi$, $n \rightarrow \infty$, в метрике $d_{\Lambda}$ эквивалентна слабой сходимости распределений $\mathscr{L}_{n} \stackrel{w}{\rightarrow} \mathscr{L}$, соответствующих преобразованиям Лапласа $\Phi_{n}$ и $\Phi$. По тем же причинам набор $\left\{d_{\Lambda}, \Lambda>1\right\}$ состоит из эквивалентных метрик. В дальнейшем в случае $b=1$ вместо векторных обозначений мы будем использовать скалярные.

Всюду далее символы $\mathbf{E}$ и $\mathbf{P}$ используются для обозначения математического ожидания и вероятности относительно меры на пространстве сред (за несколькими исключениями, которые, как мы надеемся, не приведут к недоразумениям), а символы $\mathbf{E}_{\pi}, \mathbf{P}_{\pi}$ - для обозначения условного математического ожидания и условной вероятности при фиксированной среде $\boldsymbol{\Pi}=\left(\mathbf{Q}_{\mathbf{1}}, \mathbf{Q}_{\mathbf{2}}, \ldots, \mathbf{Q}_{\mathbf{n}}, \ldots\right)$. Такого же соглашения мы будем придерживаться, рассматривая процессы с одним типом частиц.

На время мы забудем о том, что рассматривается ветвящийся процесс с двумя типами частиц и сформулируем одно утверждение, справедливое для ветвящегося процесса с одним типом частиц.

Пусть $Z_{n}$ - количество частиц в момент $n$ в ветвящемся процессе с одним типом частиц, эволюционирующем в случайной среде $\pi=\left(q_{1}, q_{2}, \ldots\right)$ и стартующем с одной частицы в момент 0 ,

$$
\begin{aligned}
\zeta_{n} & :=e^{-S_{\tau(n)}} \mathbf{P}_{\pi}\left(Z_{n}>0\right) \\
\widehat{Y}_{k} & :=\frac{Z_{k}}{\mathbf{E}_{\pi}\left[Z_{k} \mid Z_{k}>0\right]}=\frac{Z_{k} \mathbf{P}_{\pi}\left(Z_{k}>0 \mid Z_{0}=1\right)}{e^{S_{k}}} .
\end{aligned}
$$


Введем (случайные) преобразования Лапласа

$$
\Phi_{n}(\lambda)=\Phi_{n, \pi}(\lambda):=\mathbf{E}_{\pi}\left[e^{-\lambda \widehat{Y}_{n}} \mid Z_{n}>0\right]
$$

и

$$
\widetilde{\Phi}_{k, n}(\lambda)=\widetilde{\Phi}_{k, n, \pi}(\lambda):=\mathbf{E}_{\pi}\left[e^{-\lambda \widehat{Y}_{k}} \mid Z_{n}>0\right] .
$$

Пусть $\mathbf{P}$ обозначает меру, задающую случайную среду П для нашего ветвящегося процесса с одним типом частиц. Эта мера порождает соответствующую меру для условных законов распределения $\mathscr{L}_{\pi}(\cdot)=$ $\mathscr{L}(\cdot \mid \Pi=\pi) \in \mathbf{L}$, задаваемых на траекториях ветвящегося процесса. Пусть $\left\{\mathscr{L}_{N, \pi}(\cdot), N \geqslant 1\right\}$ - последовательность таких (случайных) вероятностных законов. Будем говорить, что эта последовательность слабо сходится к (случайному) вероятностному закону $\mathscr{L}_{\pi}(\cdot)$ при $N \rightarrow \infty$ и писать

$$
\mathscr{L}_{N, \pi}(\cdot) \Rightarrow \mathscr{L}_{\pi}(\cdot)
$$

если для любой неслучайной ограниченной функции $J: \mathbb{R} \rightarrow \mathbb{R}$ и любой неслучайной непрерывной функции $g: \mathbb{R} \rightarrow \mathbb{R}$

$$
\lim _{N \rightarrow \infty} \mathbf{E}\left[g\left(\int x \mathscr{L}_{N, \pi}(d x)\right)\right]=\mathbf{E}\left[g\left(\int x \mathscr{L}_{\pi}(d x)\right)\right]
$$

или, в терминах соответствующих преобразований Лапласа (если они определены), что

$$
\lim _{N \rightarrow \infty} \mathbf{E}\left[g\left(\int e^{-\lambda x} \mathscr{L}_{N, \pi}(d x)\right)\right]=\mathbf{E}\left[g\left(\int e^{-\lambda x} \mathscr{L}_{\pi}(d x)\right)\right] .
$$

Следующая теорема является комбинацией результатов, установленных в работах [9] и [10].

Теорема 1. Если выполнень условия А2 и $\mathrm{A} 3$, то

$$
\zeta_{n} \stackrel{d}{\rightarrow} \zeta
$$

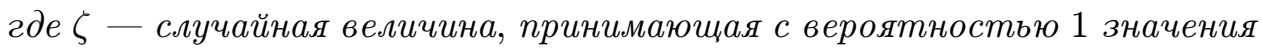
из множества $(0,1]$. Кроме того,

$$
\widetilde{\Phi}_{n t, n, \pi}(\lambda) \Rightarrow \Phi(\lambda) I\left\{\tau_{t}=\tau_{1}\right\}-\Phi^{\prime}(\lambda) I\left\{\tau_{t}<\tau_{1}\right\}, \quad n \rightarrow \infty,
$$

где случайная функиия $\Phi(\lambda)=\int_{0}^{\infty} e^{-\lambda y} \mathscr{L}(d y), \lambda \in \mathbb{R}_{+}$, является преобразованием Лапласа (случайного) вероятностного закона $\mathscr{L}$, принадлежащего множеству $\mathbf{L}^{+}$с вероятностью 1, причем $\Phi(\lambda)$ не зависит om napbl $\left(\tau_{t}, \tau_{1}\right)$. 
Наша первая цель - доказать аналог этой теоремы для процессов с двумя типами частиц, эволюционирующих в асинхронных случайных средах.

Положим

$$
\zeta_{n}^{(1)}:=\frac{\mathbf{P}_{\pi}\left(Z_{n}^{(1)}>0\right)}{e^{S_{\tau(n)}(1)}}, \quad \zeta_{n}^{(2)}:=\frac{\mathbf{P}_{\pi}\left(Z_{n}^{(2)}>0\right)}{e^{S_{\tau(n)}(2)}}=e^{S_{\mu(n)}(1)} \mathbf{P}_{\pi}\left(Z_{n}^{(2)}>0\right),
$$

где, при определенной вольности в обозначениях, $S_{\tau(n)}(i)$ и $S_{\mu(n)}(i)$ обозначают минимальное и максимальное значения случайного блуждания $\left\{S_{k}(i), 0 \leqslant k \leqslant n\right\}, i=1,2$.

В дальнейшем будет использоваться следующее соглашение: если мы снабжаем индексом $i \in\{1,2\}$ функцию или случайную величину, которые ранее были определены без индексов, мы всегда имеем ввиду соответствующую функцию или случайную величину для $i-$ го ветвящегося процесса. Например,

$$
Y_{n t}^{(1)}=\frac{Z_{n t}^{(1)}}{\mathbf{E}_{\pi} Z_{n t}^{(1)}}=Z_{n t}^{(1)} e^{-S_{n t}(1)}, \quad Y_{n t}^{(2)}=\frac{Z_{n t}^{(2)}}{\mathbf{E}_{\pi} Z_{n t}^{(2)}}=Z_{n t}^{(2)} e^{-S_{n t}(2)}=Z_{n t}^{(2)} e^{S_{n t}(1)} .
$$

Теперь мы можем сформулировать наш первый результат.

Теорема 2. Если выполнены условия $\mathrm{A} 1-\mathrm{A} 3$, то при $n \rightarrow \infty$

$$
\left(\zeta_{n}^{(1)}, \zeta_{n}^{(2)}\right) \stackrel{d}{\rightarrow}\left(\zeta^{(1)}, \zeta^{(2)}\right)
$$

где случайные величинь $\zeta^{(i)}$ независимь и с вероятностью 1 принимают значения из множества $(0,1]$. Кроме того, для $t \in(0,1]$ преобразования Лапласа

$$
\widetilde{\Phi}_{n t, n}^{(i)}\left(\lambda_{i}\right):=\mathbf{E}_{\pi}\left[e^{-\lambda_{i} \widehat{Y}_{n t}^{(i)}} \mid Z_{n}^{(i)}>0\right], \quad \lambda_{i} \in \mathbb{R}_{+}, \quad i=1,2,
$$

удовлетворяют соотношению

$$
\begin{aligned}
\left(\widetilde{\Phi}_{n t, n}^{(1)}\left(\lambda_{1}\right), \widetilde{\Phi}_{n t, n}^{(2)}\left(\lambda_{2}\right)\right) \Rightarrow & \left(\Phi^{(1)}\left(\lambda_{1}\right), \Phi^{(2)}\left(\lambda_{2}\right)\right) I\left\{\max \left\{\tau_{1}, \mu_{1}\right\}<t\right\} \\
& +\left(\Phi^{(1)}\left(\lambda_{1}\right),-\Phi^{(2) \prime}\left(\lambda_{2}\right)\right) I\left\{\tau_{1}<t<\mu_{1}\right\} \\
& +\left(-\Phi^{(1) \prime}\left(\lambda_{1}\right), \Phi^{(2)}\left(\lambda_{2}\right)\right) I\left\{\mu_{1}<t<\tau_{1}\right\} \\
& -\left(\Phi^{(1) \prime}\left(\lambda_{1}\right), \Phi^{(2) \prime}\left(\lambda_{2}\right)\right) I\left\{\min \left\{\tau_{1}, \mu_{1}\right\}>t\right\},
\end{aligned}
$$

в котором $\Phi^{(i)}\left(\lambda_{i}\right), i=1,2,-($ случайнье $)$ преобразования Лапласа собственных случайньх величин. Более того, случайные величины $\Phi^{(i)}\left(\lambda_{i}\right)$, $i=1,2$, независимы друг от друга и от $\mu_{1}, \tau_{t} u \tau_{1}$. 
3 а м е ч а н и е 1 . При $t=1$ последний член в правой части (10) равен нулю.

3 а м е ч а н и е 2. В силу (9) второе утверждение теоремы означает, нестрого говоря, что если оба типа не выродились до момента $n$, то число индивидуумов типа $i$ в момент $n t$ пропорционально величине $\exp \left\{S_{n t}(i)-S_{\tau(n t)}(i)\right\}$.

Для $s, t \in[0,1]$ положим

$$
\begin{aligned}
\varphi_{\tau(n t), n}^{(1)}(s) & :=\mathbf{E}_{\pi}\left[s_{\tau(n t)}^{Z_{(1)}^{(1)}} \mid Z^{(1)}(n)>0\right], \\
\widetilde{\Phi}_{\tau(n t), n}^{(2)}(\lambda) & :=\mathbf{E}_{\pi}\left[e^{-\lambda \widehat{Y}_{\tau(n t)}^{(2)}} \mid Z_{n}^{(2)}>0\right] .
\end{aligned}
$$

Теперь мы сформулируем утверждение, описывающее распределение числа частиц в обоих процессах в случайные моменты $\tau(n t), 0<t \leqslant 1$, при условии, что $\left\{Z_{n}^{(1)}>0, Z_{n}^{(2)}>0\right\}$.

Теорема 3. Если выполненьи условия А1-A3, то для любого $t \in$ $(0,1]$ nрu $n \rightarrow \infty$

$$
\begin{aligned}
\left(\varphi_{\tau(n t), n}^{(1)}(s), \widetilde{\Phi}_{\tau(n t), n}^{(2)}(\lambda)\right) \Rightarrow & \left(\varphi^{(1)}(s), \Phi^{(2)}(\lambda)\right) I\left\{\mu_{1}<\tau_{t}=\tau_{1}\right\} \\
& +\left(\varphi^{(1)}(s),-\Phi^{(2) \prime}(\lambda)\right) I\left\{\mu_{1}>\tau_{t}=\tau_{1}\right\} \\
& +\left(\varphi^{*}(s), \Phi(\lambda)\right) I\left\{\mu_{1}<\tau_{t}<\tau_{1}\right\} \\
& +\left(\varphi^{*}(s),-\Phi^{(2) \prime}(\lambda)\right) I\left\{\tau_{t}<\min \left\{\tau_{1}, \mu_{1}\right\}\right\}
\end{aligned}
$$

где $\varphi^{(1)}(s), \varphi^{*}(s)$ - (случайнье) производящие функиии собственньи распределений с носителями во множестве $\{1,2, \ldots\}$, a $\Phi^{(2)}(\lambda)$ имеет такое же распределение, как и в теореме 2 . Кроме того, $\varphi^{(1)}(s), \varphi^{*}(s)$ и $\Phi^{(2)}(\lambda)$ взаимно независимы и независимы от $\mu_{1}, \tau_{t}, \tau_{1}$.

3 а м е ч а н и е 3 . При $t=1$ только первые два слагаемых в правой части (12) отличны от нуля.

3 а м е ч а н и е 4. Теорема 3 допускает следующую нестрогую трактовку. Если оба типа не вымерли к моменту $n \rightarrow \infty$, то в моменты $\tau(n t)$ количество частиц первого типа в процессе мало (предельное распределение случайной величины $Z_{\tau(n t)}^{(1)}$ дискретно), в то время как размер популяции частиц второго типа весьма велик (пропорционален $\exp \left\{S_{\mu(\tau(n t))}-S_{\tau(n t)}\right\}$, где величина $S_{\mu(\tau(n t))}-S_{\tau(n t)}$ имеет порядок $\sqrt{n}$, если $\left.\mathbf{E} X_{1}(1)<\infty\right)$. Само собой разумеется, что сходная картина имеет место в моменты $\mu(n t)$, когда размер популяции частиц первого типа имеет порядок $\exp \left\{S_{\mu(n t)}-S_{\tau(\mu(n t))}\right\}$, в то время как частиц второго типа мало. С некой долей вольности это явление можно трактовать как осцилляцию размеров популяций хищников и жертв в моделях типа хищникжертва. А именно, если в каком-либо поколении среднее число потомков 
у хищников велико $\left(X_{k}(1) \gg 1\right)$, то среднее число потомков жертв, достигших репродуктивного возраста, мало $\left(X_{k}(2)=-X_{k}(1) \ll-1\right)$.

Можно поставить задачу доказательства многомерных версий упомянутых ранее теорем. K сожалению, даже формулировка такого рода обобщений занимает много места. В связи с этим мы ограничимся теоремой, описывающей совместное распределение числа частиц обоих типов в моменты $0<n t_{1}<\cdots<n t_{d}=n, d \geqslant 2$. Читатель может найти соответствующее утверждение и его доказательство в разд. 3.

2. Вспомогательные результаты. Рассмотрим сначала ветвящиеся процессы в случайной среде с одним типом частиц.

Можно проверить (см., например, лемму 1 работы [10]), что для любого осциллирующего случайного блуждания

$$
\begin{array}{ll}
\mathbf{E}[U(x-X) ; X-x<0]=U(x), & x \in \mathbb{R}_{+}, \\
\mathbf{E}[V(x+X) ; X+x \geqslant 0]=V(x), & x \in \mathbb{R}_{+} .
\end{array}
$$

При помощи функций восстановления $U$ и $V$ мы построим две вероятностные меры $\mathbf{P}^{+}$and $\mathbf{P}^{-}$, используя следующую процедуру.

Рассмотрим фильтрацию $\mathscr{F}=\left(\mathscr{F}_{n}, n \geqslant 0\right)$, где сигма-алгебра $\mathscr{F}_{n}=$ $\sigma\left(Q_{1}, \ldots, Q_{n}, Z_{0}, \ldots, Z_{n}\right)$. Таким образом, случайное блуждание $\left\{S_{k}, k \geqslant\right.$ $0\}$ согласовано с фильтрацией $\mathscr{F}$, причем $X_{n+1}$ (так же, как и $Q_{n+1}$ ) не зависит от сигма-алгебры $\mathscr{F}_{n}$ при всех $n \geqslant 0$. Далее мы строим вероятностные меры $\mathbf{P}_{x}^{-}, x \leqslant 0$, и $\mathbf{P}_{y}^{+}, y \geqslant 0$, которые для каждого $n$ и каждой ограниченной и $\mathscr{F}_{n}$-измеримой случайной величины $R_{n}$ задаются равенствами

$$
\mathbf{E}_{x}^{-}\left[R_{n}\right]:=\frac{1}{U(-x)} \mathbf{E}_{x}\left[R_{n} U\left(-S_{n}\right) ; M_{n}<0\right]
$$

и

$$
\mathbf{E}_{y}^{+}\left[R_{n}\right]:=\frac{1}{V(y)} \mathbf{E}_{y}\left[R_{n} V\left(S_{n}\right) ; L_{n} \geqslant 0\right] .
$$

Используя (13)-(14), нетрудно проверить (см. [10], где этот вопрос детально обсуждается), что меры, заданные таким образом, согласованы по $n$.

С мерами $\mathbf{P}_{x}^{-}, x \leqslant 0$, и $\mathbf{P}_{y}^{+}, y \geqslant 0$, мы свяжем еще одну меру $\widehat{\mathbf{P}}_{x, y}$. Пусть $\left\{Q_{k}^{+}, k \geqslant 0\right\}$ и $\left\{Q_{k}^{-}, k \geqslant 0\right\}$ - две независимых последовательности случайных сред, построенных при помощи исходной меры $\mathbf{P}$, и пусть $\left\{Z_{k}^{-}, k \geqslant 0\right\}$ и $\left\{Z_{k}^{+}, k \geqslant 0\right\}-$ соответствующие ветвящиеся процессы, а $\left\{S_{k}^{-}, k \geqslant 0\right\}$ и $\left\{S_{k}^{+}, k \geqslant 0\right\}-$ ассоциированные с этими процессами случайные блуждания.

Рассмотрим теперь фильтрацию $\widehat{\mathscr{F}}=\left(\widehat{\mathscr{F}}_{r, n}, r, n \geqslant 0\right)$, где
$\widehat{\mathscr{F}}_{r, n}=\mathscr{F}_{r}^{-} \times \mathscr{F}_{n}^{+}$с $\mathscr{F}_{r}^{-}=\sigma\left(Q_{1}^{-}, \ldots, Q_{r}^{-}, Z_{0}^{-}, \ldots, Z_{r}^{-}\right)$и $\mathscr{F}_{n}^{+}=$ 
$\sigma\left(Q_{1}^{+}, \ldots, Q_{n}^{+}, Z_{0}^{+}, \ldots, Z_{n}^{+}\right)$. Для каждой ограниченной и $\widehat{\mathscr{F}}_{r, n}$-измеримой случайной величины $R_{r, n}$ и $x \leqslant 0, y \geqslant 0$ положим

$$
\begin{aligned}
\widehat{\mathbf{E}}_{x, y}\left[R_{r, n}\right] & =\left(\mathbf{E}_{x}^{-} \times \mathbf{E}_{y}^{+}\right)\left[R_{r, n}\right] \\
& :=\frac{1}{U(-x) V(y)}\left(\mathbf{E}_{x} \times \mathbf{E}_{y}\right)\left[R_{r, n} U\left(-S_{r}^{-}\right) V\left(S_{n}^{+}\right) ; M_{r}^{-}<0, L_{n}^{+} \geqslant 0\right] .
\end{aligned}
$$

Лемма 1 (см. [10]). Пусть выполнено условие А2, и пусть $\left\{W_{r, n}\right.$, $r, n \geqslant 0\}$ - схема серий равномерно ограниченньх случайных величин, согласованньх с фильтрачией $\widehat{\mathscr{F}}$. Тогда для любых фиксированных $k, l$

$$
\lim _{\min (r, n) \rightarrow \infty} \mathbf{E}\left[W_{k, l} \mid M_{r}^{-}<0, L_{n}^{+} \geqslant 0\right]=\widehat{\mathbf{E}}\left[W_{k, l}\right] .
$$

Если $W_{r, n} \rightarrow W_{\infty}$ при $\min (r, n) \rightarrow \infty \widehat{\mathbf{P}}-$ п.н. для некоторой случайной величинь $W_{\infty}$, то

$$
\lim _{\min (r, n) \rightarrow \infty} \mathbf{E}\left[W_{r, n} \mid M_{r}^{-}<0, L_{n}^{+} \geqslant 0\right]=\widehat{\mathbf{E}}\left[W_{\infty}\right] .
$$

Следствие 1. Пусть выполнено условие А2, и пусть $\left\{W_{r, n}, r, n \geqslant\right.$ 0\} - схема серий ограниченных случайных величин, согласованных $c$ фильтраиией $\widehat{\mathscr{F}}$. Если $W_{r, n} \rightarrow W_{\infty}$ при $\min (r, n) \rightarrow \infty \widehat{\mathbf{P}}$-n.н. для собственной случайной величины $W_{\infty}$, то при $\min (r, n) \rightarrow \infty$

$$
\mathscr{L}\left(W_{r, n} \mid M_{r}^{-}<0, L_{n}^{+} \geqslant 0\right) \stackrel{w}{\rightarrow} \mathscr{L}\left(W_{\infty}\right) .
$$

Д о к а з а т е л ь с т в о. В силу леммы 1 для любого $t \in(-\infty,+\infty)$

$$
\lim _{\min (r, n) \rightarrow \infty} \mathbf{E}\left[e^{i t W_{r, n}} \mid M_{r}^{-}<0, L_{n}^{+} \geqslant 0\right]=\widehat{\mathbf{E}}\left[e^{i t W_{\infty}}\right],
$$

что и завершает доказательство.

Нам понадобится следующее утверждение, доказанное в лемме 2 работы [10].

Лемма 2. Если выполнено условие $\mathrm{A} 2$, то для любого $x \in \mathbb{R}_{+} n р и$ $n \rightarrow \infty$

$$
\mathbf{P}\left(L_{n} \geqslant-x\right) \sim \frac{V(x)}{n^{1-\rho} l_{1}(n)}, \quad \mathbf{P}\left(M_{n}<x\right) \sim \frac{U(x)}{n^{\rho} l_{2}(n)},
$$

где $l_{1}(n), l_{2}(n)$ - функиии медленно меняюшиеся на бесконечности такuе, ито

$$
l_{1}(n) l_{2}(n) \sim \pi / \sin \pi \rho, \quad n \rightarrow \infty .
$$

Кроме того, найдется константа $C>0$ такая, ито для всех $x \in \mathbb{R}_{+}$

$$
\mathbf{P}\left(L_{n} \geqslant-x\right) \leqslant C \frac{V(x)}{n^{1-\rho} l_{1}(n)}, \quad \mathbf{P}\left(M_{n}<x\right) \leqslant C \frac{U(x)}{n^{\rho} l_{2}(n)} .
$$


Теперь мы установим несколько вспомогательных результатов.

Положим

$$
\begin{aligned}
& f_{j, n}(s):=f_{j+1}\left(f_{j+2}\left(\ldots\left(f_{n}(s)\right) \ldots\right)\right), 0 \leqslant j \leqslant n-1, \\
& f_{n, n}(s):=s \\
& f_{n, j}(s):=f_{n}\left(f_{n-1}\left(\ldots\left(f_{j+1}(s)\right) \ldots\right)\right), \quad n \geqslant j \geqslant 1 .
\end{aligned}
$$

Обозначим

$$
\Delta_{n}\left(m, k ; s, s_{1}\right):=\frac{f_{0, m}\left(s f_{m, n}\left(s_{1}\right)\right)-f_{0, m}\left(s f_{m, m+k}\left(s_{1}\right)\right)}{e^{S_{m}}} .
$$

Лемма 3. Для любых фиксированных $0 \leqslant s, s_{1} \leqslant 1$ и любого $\varepsilon>0$

$$
\lim _{k \rightarrow \infty} \lim \sup _{n \rightarrow \infty} \mathbf{P}\left(\left|\Delta_{n}\left(m, k ; s, s_{1}\right)\right|>\varepsilon \mid \tau(n)=m\right)=0 .
$$

Д о к а з а т ел ь с т в о. Имеем

$$
\left|\Delta_{n}\left(m, k ; s, s_{1}\right)\right| \leqslant\left|f_{m, n}\left(s_{1}\right)-f_{m, m+k}\left(s_{1}\right)\right| .
$$

Следовательно, при $n \geqslant m+k$

$$
\begin{aligned}
\mathbf{P}\left(\left|\Delta_{n}\left(m, k ; s, s_{1}\right)\right|>\varepsilon \mid \tau(n)=m\right) \\
\leqslant \varepsilon^{-1} \mathbf{E}\left[\left|f_{m, n}\left(s_{1}\right)-f_{m, m+k}\left(s_{1}\right)\right| \mid \tau(n)=m\right] \\
\quad=\varepsilon^{-1} \mathbf{E}\left[\left|f_{m, n}\left(s_{1}\right)-f_{m, m+k}\left(s_{1}\right)\right| \mid L_{n-m} \geqslant 0\right] .
\end{aligned}
$$

Согласно теореме 5 из [2] для любого $s_{1} \in[0,1]$ существует предел

$$
\lim _{n \rightarrow \infty} f_{0, n}\left(s_{1}\right):=f_{0, \infty}\left(s_{1}\right) .
$$

Отсюда, устремляя $n \rightarrow \infty$, получаем при помощи леммы 1 , что

$\lim \sup _{n \rightarrow \infty} \mathbf{P}\left(\left|\Delta_{n}\left(m, k ; s, s_{1}\right)\right|>\varepsilon \mid \tau(n)=m\right) \leqslant \varepsilon^{-1} \mathbf{E}^{+}\left[\left|f_{0, \infty}\left(s_{1}\right)-f_{0, k}\left(s_{1}\right)\right|\right]$.

Устремляя теперь $k$ к бесконечности и используя теорему о мажорируемой сходимости, приходим к желаемому утверждению.

Следующее утверждение показывает, что аналог предыдущей леммы справедлив и при подходящей скорости стремления $s=s(n)$ к единице.

Лемма 4. Для любых $\lambda \in \mathbb{R}_{+} u \varepsilon>0$

$$
\begin{gathered}
\lim _{k \rightarrow \infty} \lim _{n \rightarrow \infty} \mathbf{P}\left(\left|\frac{f_{0, n}\left(\exp \left\{-\lambda e^{S_{m}-S_{n}}\right\}\right)-f_{0, m+k}\left(\exp \left\{-\lambda e^{S_{m}-S_{k}}\right\}\right)}{e^{S_{m}}}\right|\right. \\
>\varepsilon \mid \tau(n)=m)=0 .
\end{gathered}
$$


Д о к а з а т е л ь с т в о. Так же, как и в предыдущей лемме, получаем

$$
\begin{aligned}
& \mathbf{P}\left(\left|\frac{f_{0, n}\left(\exp \left\{-\lambda e^{S_{m}-S_{n}}\right\}\right)-f_{0, m+k}\left(\exp \left\{-\lambda e^{S_{m}-S_{k}}\right\}\right)}{e^{S_{m}}}\right|>\varepsilon \mid \tau(n)=m\right) \\
& \quad \leqslant \varepsilon^{-1} \mathbf{E}\left[\left|f_{0, n-m}\left(\exp \left\{-\lambda e^{-S_{n-m}}\right\}\right)-f_{0, k}\left(\exp \left\{-\lambda e^{-S_{k}}\right\}\right)\right| \mid L_{n-m} \geqslant 0\right] .
\end{aligned}
$$

Поскольку

$$
f_{0, r}\left(\exp \left\{-\lambda e^{-S_{r}}\right\}\right)=\mathbf{E}_{\pi}\left[\exp \left\{-\lambda Z_{r} e^{-S_{r}}\right\}\right]
$$

и $\left\{Z_{r} e^{-S_{r}}, r \geqslant 0\right\}$ является неотрицательным мартингалом, то для любого $\lambda \in \mathbb{R}_{+}$предел

$$
F_{\pi}(\lambda):=\lim _{r \rightarrow \infty} f_{0, r}\left(\exp \left\{-\lambda e^{-S_{r}}\right\}\right)
$$

существует $\mathbf{P}^{+}$-п.н. (и, следовательно, $\widehat{\mathbf{P}}$-п.н.). Отсюда, устремляя $n$ к бесконечности, заключаем при помощи леммы 1 , что

$$
\begin{aligned}
\lim _{n \rightarrow \infty} & \mathbf{E}\left[\left|f_{0, n-m}\left(\exp \left\{-\lambda e^{-S_{n-m}}\right\}\right)-f_{0, k}\left(\exp \left\{-\lambda e^{-S_{k}}\right\}\right)\right| \mid L_{n-m} \geqslant 0\right] \\
& =\mathbf{E}^{+}\left[\left|F_{\pi}(\lambda)-f_{0, k}\left(\exp \left\{-\lambda e^{-S_{k}}\right\}\right)\right|\right] .
\end{aligned}
$$

Из этого равенства и (17), устремляя $k$ к бесконечности, получаем утверждение леммы.

Обозначим

$$
\Delta\left(m, k ; s, s_{1}\right):=\frac{1-f_{m-k, m}\left(s f_{m, m+k}\left(s_{1}\right)\right)}{e^{S_{m}-S_{m-k}}}-\frac{1-f_{0, m}\left(s f_{m, m+k}\left(s_{1}\right)\right)}{e^{S_{m}}} .
$$

Лемма 5. Для любого $\varepsilon>0$

$$
\lim _{k \rightarrow \infty} \limsup _{\min (m, n-m) \rightarrow \infty} \mathbf{P}\left(\sup _{0 \leqslant s, s_{1} \leqslant 1}\left|\Delta\left(m, k ; s, s_{1}\right)\right|>\varepsilon \mid \tau(n)=m\right)=0 .
$$

Д ок а з а т ель с т в о. Имеем

$$
\begin{aligned}
\left|\Delta\left(m, k ; s, s_{1}\right)\right|= & \frac{1-f_{m-k, m}\left(s f_{m, m+k}\left(s_{1}\right)\right)}{e^{S_{m}-S_{m-k}}} \frac{1-f_{0, m}\left(s f_{m, m+k}\left(s_{1}\right)\right)}{e^{S_{m}}} \\
& \times\left|\frac{e^{S_{m}}}{1-f_{0, m}\left(s f_{m, m+k}\left(s_{1}\right)\right)}-\frac{e^{S_{m}-S_{m-k}}}{1-f_{m-k, m}\left(s f_{m, m+k}\left(s_{1}\right)\right)}\right| \\
\leqslant & \left|\frac{e^{S_{m}}}{1-f_{0, m}\left(s f_{m, m+k}\left(s_{1}\right)\right)}-\frac{e^{S_{m}-S_{m-k}}}{1-f_{m-k, m}\left(s f_{m, m+k}\left(s_{1}\right)\right)}\right| .
\end{aligned}
$$

Положим

$$
\chi_{j}(s):=\frac{1}{1-f_{j}(s)}-\frac{1}{f_{j}^{\prime}(1)(1-s)}, \quad 0 \leqslant s \leqslant 1 .
$$


Известно (см., [6]), что для $0 \leqslant l \leqslant q-1$

$$
\frac{1}{1-f_{l, q}(s)}=\frac{e^{-S_{q}+S_{l}}}{1-s}+\sum_{j=l}^{q-1} \eta_{j, q}(s) e^{-S_{j}+S_{l}}
$$

где

$$
\eta_{j, n}(s):=\chi_{j+1}\left(f_{j+1, n}(s)\right) \leqslant \eta_{j+1}:=\frac{f_{j+1}^{\prime \prime}(1)}{\left(f_{j+1}^{\prime}(1)\right)^{2}} .
$$

Используя это представление, получаем

$$
\begin{aligned}
& \left|\frac{e^{S_{m}}}{1-f_{0, m}\left(s f_{m, m+k}\left(s_{1}\right)\right)}-\frac{e^{S_{m}-S_{m-k}}}{1-f_{m-k, m}\left(s f_{m, m+k}\left(s_{1}\right)\right)}\right| \\
& \quad=\sum_{j=0}^{m-k-1} \eta_{j, m}\left(s f_{m, m+k}\left(s_{1}\right)\right) e^{S_{m}-S_{j}} \leqslant \sum_{j=0}^{m-k-1} \eta_{j+1} e^{S_{m}-S_{j}} .
\end{aligned}
$$

Отсюда, в силу свойства дуальности для случайных блужданий, заключаем, что

$$
\begin{aligned}
& \mathbf{P}\left(\sup _{0 \leqslant s, s_{1} \leqslant 1}\left|\Delta\left(m, k ; s, s_{1}\right)\right|>\varepsilon \mid \tau(n)=m\right) \\
& \quad \leqslant \mathbf{P}\left(\sum_{j=0}^{m-k-1} \eta_{j} e^{S_{m}-S_{j}}>\varepsilon \mid \tau(n)=m\right)=\mathbf{P}\left(\sum_{j=k}^{m} \eta_{j} e^{S_{j}}>\varepsilon \mid M_{m}<0\right) .
\end{aligned}
$$

Поскольку

$$
\sum_{j=1}^{\infty} \eta_{j} e^{S_{j}}<\infty \quad \mathbf{P}^{-} \text {-п.н. }
$$

согласно лемме 6 работы [10], то соотношение (18) следует из леммы 1.

Следствие 2. Для любых фиксированных $0 \leqslant s, s_{1} \leqslant 1$ и любого $\varepsilon>0$

$$
\begin{aligned}
\lim _{k \rightarrow \infty} \lim _{n \rightarrow \infty} \sup _{n \rightarrow \infty} \mathbf{P}\left(\mid \frac{1-f_{\tau(n)-k, \tau(n)}\left(s f_{\tau(n), \tau(n)+k}\left(s_{1}\right)\right)}{e^{S_{\tau(n)}-S_{\tau(n)-k}}}\right. & \\
& \left.-\frac{1-f_{0, \tau(n)}\left(s f_{\tau(n), n}\left(s_{1}\right)\right)}{e^{S_{\tau(n)}}} \mid>\varepsilon\right)=0 .
\end{aligned}
$$

Кроме того,

$$
\lim _{k \rightarrow \infty} \lim \sup _{n \rightarrow \infty} \mathbf{P}\left(\left|\frac{e^{S_{\tau(n)-k}}\left(1-f_{\tau(n)-k, \tau(n)+k}(0)\right)}{1-f_{0, n}(0)}-1\right|>\varepsilon\right)=0 .
$$

Д о к а з а т е л ь с т в о. Для доказательства справедливости первого утверждения достаточно воспользоваться формулой полной вероятности, леммами 3,5 и обобщенным законом арксинуса для $\tau(n)$. Второе утверждение является следствием первого утверждения при $s=1, s_{1}=0$ и соотношения (7). 
Следствие 3. Для любых $s \in[0,1] u \varepsilon>0$

$$
\lim _{k \rightarrow \infty} \lim \sup _{n \rightarrow \infty} \mathbf{P}\left(\left|\frac{1-f_{\tau(n)-k, \tau(n)}(s)}{e^{S_{\tau(n)}-S_{\tau(n)-k}}}-\frac{1-f_{0, \tau(n)}(s)}{e^{S_{\tau(n)}}}\right|>\varepsilon\right)=0 .
$$

Кроме того,

$$
\lim _{k \rightarrow \infty} \lim _{n \rightarrow \infty} \mathbf{P}\left(\left|\frac{e^{S_{\tau(n)-k}}\left(1-f_{\tau(n)-k, \tau(n)}(s)\right)}{1-f_{0, \tau(n)}(s)}-1\right|>\varepsilon\right)=0 .
$$

Д о к а з а т е л ь с т в о. Для доказательства первого утверждения достаточно использовать оценку

$$
\left|\frac{e^{S_{m}}}{1-f_{0, m}(s)}-\frac{e^{S_{m}-S_{m-k}}}{1-f_{m-k, m}(s)}\right| \leqslant \sum_{j=0}^{m-k-1} \eta_{j+1} e^{S_{m}-S_{j}},
$$

равенство

$$
\mathscr{L}\left(\sum_{j=0}^{m-k-1} \eta_{j+1} e^{S_{m}-S_{j}} \mid \tau(m)=m\right)=\mathscr{L}\left(\sum_{j=k}^{m-1} \eta_{j} e^{S_{j}} \mid M_{m}<0\right),
$$

сходимость (19), лемму 1 и обобщенный закон арксинуса. Второе утверждение леммы является следствием первого утверждения, равенства

$$
\mathscr{L}\left(\frac{e^{S_{\tau(n)}-S_{\tau(n)-k}}}{1-f_{\tau(n)-k, \tau(n)}(s)} \mid \tau(n)=m\right)=\mathscr{L}\left(\frac{1}{1-s}+\sum_{j=0}^{k-1} \eta_{j, m}(s) e^{S_{j}} \mid M_{m}<0\right),
$$

обобщенного закона арксинуса, оценки (19) и леммы 1.

В дальнейшем мы будем понимать выражения вида $n t, n t_{1}$ и так далее, как $[n t],\left[n t_{1}\right]$, где $[x]$ - целая часть числа $x$.

Пусть $L^{+}$и $L^{-}$- неотрицательный и отрицательный меандры строго устойчивого процесса Леви с параметром $\alpha$.

Лемма 6. Пусть выполнено условие А2. Тогда для последовательности $c_{n}$, заданной соотношением (1), и для любого $x \in \mathbb{R}_{+}$при $n \rightarrow \infty$

$$
\begin{aligned}
& \mathscr{L}\left(\left(\frac{S_{n t}}{c_{n}}\right)_{0 \leqslant t \leqslant 1} \mid L_{n} \geqslant-x\right) \Longrightarrow \mathscr{L}\left(L^{+}\right), \\
& \mathscr{L}\left(\left(\frac{S_{n t}}{c_{n}}\right)_{0 \leqslant t \leqslant 1} \mid M_{n}<x\right) \Longrightarrow \mathscr{L}\left(L^{-}\right),
\end{aligned}
$$

где символ $\Longrightarrow$ обозначает сходимость в пространстве $D[0,1]$ функиий без разрывов второго рода, оснащенном метрикой Скорохода.

Д о к а з а т е л ь с т в о. Первое утверждение - это лемма 2.3 в [1]. Доказательство второго утверждения может быть получено почти дословным повторением доказательства упомянутой леммы с естественными изменениями.

Положим $\bar{\mu}(n):=\min \left\{0 \leqslant i \leqslant n: S_{i}=\max \left(0, M_{n}\right)\right\}$. 
Лемма 7. Если выполнено условие А2 $c \alpha \rho<1$, то найдется константа $C_{\alpha}>0$ такая, ито для всех $n \geqslant 1$ и всех $N \geqslant 1$

$$
\begin{aligned}
\mathbf{P}\left(S_{n} \geqslant N c_{n}, L_{n} \geqslant 0, \bar{\mu}(n)=n\right) & \leqslant C_{\alpha} \mathbf{P}\left(S_{n} \geqslant N c_{n}\right) \mathbf{P}^{2}\left(L_{n} \geqslant 0\right) \\
& \leqslant C_{\alpha}^{2} n \mathbf{P}\left(X \geqslant N c_{n}\right) \mathbf{P}^{2}\left(L_{n} \geqslant 0\right) .
\end{aligned}
$$

Д ок аз а т е ль с т в о. Для $0 \leqslant k \leqslant n-[n / 2]$ положим $S_{k}^{\prime}:=$ $S_{k+[n / 2]}-S_{[n / 2]}$. Введем события

$$
\mathscr{B}_{\min }(n):=\left\{\min _{0 \leqslant i \leqslant[n / 2]} S_{i} \geqslant 0, S_{[n / 2]}+\min _{0 \leqslant k \leqslant n-[n / 2]} S_{k}^{\prime} \geqslant 0\right\}
$$

и

$$
\begin{aligned}
& \mathscr{B}_{\max }(n):=\left\{\max _{0 \leqslant i \leqslant[n / 2]} S_{i}<S_{[n / 2]}+S_{n-[n / 2]}^{\prime}, S_{[n / 2]}\right. \\
&\left.+\max _{0 \leqslant k \leqslant n-[n / 2]-1} S_{k}^{\prime}<S_{[n / 2]}+S_{n-[n / 2]}^{\prime}\right\} .
\end{aligned}
$$

Имеем

$$
\begin{aligned}
\mathbf{P}( & \left.S_{n} \geqslant N c_{n}, L_{n} \geqslant 0, \bar{\mu}(n)=n\right) \\
= & \mathbf{P}\left(S_{[n / 2]}+S_{n-[n / 2]}^{\prime} \geqslant N c_{n}, \mathscr{B}_{\min }(n), \mathscr{B}_{\max }(n)\right) \\
\leqslant & \mathbf{P}\left(S_{[n / 2]} \geqslant 2^{-1} N c_{n}, \mathscr{B}_{\min }(n), \mathscr{B}_{\max }(n)\right) \\
& +\mathbf{P}\left(S_{n-[n / 2]}^{\prime} \geqslant 2^{-1} N c_{n}, \mathscr{B}_{\min }(n), \mathscr{B}_{\max }(n)\right) \\
\leqslant & \mathbf{P}\left(S_{[n / 2]} \geqslant 2^{-1} N c_{n}, L_{[n / 2]} \geqslant 0, \max _{0 \leqslant k \leqslant n-[n / 2]} S_{k}^{\prime} \leqslant S_{n-[n / 2]}^{\prime}\right) \\
& +\mathbf{P}\left(S_{n-[n / 2]}^{\prime} \geqslant 2^{-1} N c_{n}, L_{[n / 2]} \geqslant 0, \max _{0 \leqslant k \leqslant n-[n / 2]} S_{k}^{\prime} \leqslant S_{n-[n / 2]}^{\prime}\right) \\
= & \mathbf{P}\left(S_{[n / 2]} \geqslant 2^{-1} N c_{n}, L_{[n / 2]} \geqslant 0\right) \mathbf{P}\left(L_{n-[n / 2]} \geqslant 0\right) \\
& +\mathbf{P}\left(S_{n-[n / 2]} \geqslant 2^{-1} N c_{n}, L_{n-[n / 2]} \geqslant 0\right) \mathbf{P}\left(L_{[n / 2]} \geqslant 0\right) .
\end{aligned}
$$

В силу теоремы 1 из [4] с $\alpha \rho<1$, для любого $\varepsilon>0$ можно найти $n(\varepsilon)$ и $N(\varepsilon)$ такие, что для всех $n \geqslant n(\varepsilon)$ и всех $N \geqslant N(\varepsilon)$

$$
\rho \mathbf{P}\left(S_{n}>N c_{n} \mid L_{n} \geqslant 0\right) \leqslant(1+\varepsilon) \mathbf{P}\left(S_{n}>N c_{n}\right) \leqslant(1+2 \varepsilon) n \mathbf{P}\left(X>N c_{n}\right) .
$$

(В действительности указанная теорема доказана в [4] при условии $\left\{L_{n}>0\right\}$. Однако анализ доказательства упомянутой теоремы показывает, что это условие можно заменить условием $\left\{L_{n} \geqslant 0\right\}$.)

Теперь необходимое утверждение следует из $(23),(22),(3)$ и свойств правильно меняющихся функций при помощи простых оценок и преобразований. 
Обозначим $B_{t}, 0 \leqslant t \leqslant 1-$ стандартное броуновское движение, и пусть

$\mathfrak{l}:=\inf _{0 \leqslant t \leqslant 1} B_{t}, \quad \mathfrak{m}:=\max _{0 \leqslant t \leqslant 1} B_{t}, \quad H_{\mathfrak{l}}=\inf _{0 \leqslant t \leqslant 1}\left\{B_{t}=\mathfrak{l}\right\}, \quad H_{\mathfrak{m}}:=\sup _{0 \leqslant t \leqslant 1}\left\{B_{t}=\mathfrak{m}\right\}$.

Лемма 8. Совместное распределение случайных величин $\left(\mathfrak{l}, H_{\mathfrak{l}}\right.$, $\left.\mathfrak{m}, H_{\mathfrak{m}}\right)$ имеет абсолютно непрерывную плотность.

Д о к а з а т е л ь с т в о. Смотрите формулу 1.28.4 монографии [3].

Лемма 9. Если выполнено условие А2, то для любого $t \in(0,1]$

$$
\lim _{\varepsilon \rightarrow 0} \lim \sup _{n \rightarrow \infty} \mathbf{P}(|\tau(n t)-\mu(n)| \leqslant n \varepsilon)=0 .
$$

Д о к а з а т е ль с т в о. Если $\mathbf{E} X^{2}<\infty$, то необходимое утверждение легко следует из принципа инвариантности для случайных блужданий и леммы 8.

Поэтому мы будем предполагать, что $\alpha \rho<1$, и рассмотрим лишь случай $t=1$. Случай $0<t<1$ может быть проанализирован аналогичным образом.

Обозначим $L(t), 0 \leqslant t \leqslant 1$, строго устойчивый процесс Леви с параметром $\alpha$. Из принципа инвариантности для процессов Леви вытекает, что для любого $\delta \in(0,1)$

$$
\begin{gathered}
\lim _{\varepsilon \downarrow 0} \lim \sup _{n \rightarrow \infty} \mathbf{P}\left(S_{\mu(n)}-S_{\tau(n)} \leqslant \varepsilon^{(1-\delta) / \alpha} c_{n}\right) \\
\leqslant \lim _{\varepsilon \downarrow 0} \lim _{n \rightarrow \infty} \mathbf{P}\left(S_{\mu(n)} \leqslant \varepsilon^{(1-\delta) / \alpha} c_{n}\right) \\
=\lim _{\varepsilon \downarrow 0} \mathbf{P}\left(\max _{0 \leqslant u \leqslant 1} L(t) \leqslant \varepsilon^{(1-\delta) / \alpha}\right)=0 .
\end{gathered}
$$

Введем событие

$$
\mathscr{A}(n, \varepsilon):=\left\{\sup _{-n \varepsilon \leqslant j \leqslant n \varepsilon}\left(S_{\tau(n)+j}-S_{\tau(n)}\right) \geqslant \varepsilon^{(1-\delta) / \alpha} c_{n}\right\} .
$$

Ясно, что

$$
\begin{aligned}
& \mathbf{P}\left(|\tau(n)-\mu(n)| \leqslant n \varepsilon, S_{\mu(n)}-S_{\tau(n)} \geqslant \varepsilon^{(1-\delta) / \alpha} c_{n}\right) \\
& \quad=\mathbf{P}(|\tau(n)-\mu(n)| \leqslant n \varepsilon, \mathscr{A}(n, \varepsilon))=R_{1}(n, \varepsilon)+R_{2}(n, \varepsilon),
\end{aligned}
$$

где

$$
R_{1}(n, \varepsilon):=\mathbf{P}(|\tau(n)-\mu(n)| \leqslant n \varepsilon, \mathscr{A}(n, \varepsilon), \tau(n) \notin[2 n \varepsilon, n(1-2 \varepsilon)])
$$

и

$$
R_{2}(n, \varepsilon):=\mathbf{P}(|\tau(n)-\mu(n)| \leqslant n \varepsilon, \mathscr{A}(n, \varepsilon), \tau(n) \in[2 n \varepsilon, n(1-2 \varepsilon)]) .
$$


В силу обобщенного закона арксинуса

$$
\lim _{\varepsilon \downarrow 0} \lim \sup _{n \rightarrow \infty} R_{1}(n, \varepsilon) \leqslant \lim _{\varepsilon \downarrow 0} \lim \sup _{n \rightarrow \infty} \mathbf{P}(\tau(n) \notin[2 n \varepsilon, n(1-2 \varepsilon)])=0 .
$$

Далее запишем

$$
R_{2}(n, \varepsilon)=R_{2-}(n, \varepsilon)+R_{2+}(n, \varepsilon),
$$

где знак «-» означает, что мы рассматриваем такие траектории случайного блуждания, в которых $\tau(n)<\bar{\mu}(n)$, а знак «+» означает, что мы рассматриваем траектории случайного блуждания, на которых указанные моменты расположены в обратном порядке.

Покажем, что

$$
\lim _{\varepsilon \downarrow 0} \lim \sup _{n \rightarrow \infty} R_{2-}(n, \varepsilon)=0 .
$$

С этой целью для фиксированных $k$ и $i>1$ рассмотрим событие $\{\tau(n)=$ $k, \bar{\mu}(n)=k+i\}$ и введем три случайных блуждания

$$
\begin{gathered}
S_{j}^{\prime}:=S_{k}-S_{k-j}, 0 \leqslant j \leqslant k ; \quad S_{j}^{\prime \prime}:=S_{k+j}-S_{k}, 0 \leqslant j \leqslant i ; \\
S_{j}^{\prime \prime \prime}:=S_{k+i+j}-S_{k+i}, 0 \leqslant j \leqslant n-k-i .
\end{gathered}
$$

Используя формулу полной вероятности и свойство дуальности случайных блужданий, имеем при $k \in[2 n \varepsilon, n(1-2 \varepsilon)]$ и $0 \leqslant i \leqslant n \varepsilon$

$$
\begin{aligned}
& \mathbf{P}(\tau(n)=k, \bar{\mu}(n)=k+i, \mathscr{A}(n, \varepsilon)) \\
& =\iint_{y-x \geqslant \varepsilon^{(1-\delta) / \alpha} c_{n}} \mathbf{P}\left(\tau(n)=k, S_{k} \in d x, \bar{\mu}(n)=k+i, S_{k+i} \in d y\right) \\
& =\iint_{y-x \geqslant \varepsilon^{(1-\delta) / \alpha} c_{n}} \mathbf{P}\left(S_{k}^{\prime} \in d x, L_{k}^{\prime}>x-y, M_{k}^{\prime}<0\right) \\
& \times \mathbf{P}\left(S_{i}^{\prime \prime} \in d(y-x), L_{i}^{\prime \prime} \geqslant 0, \bar{\mu}^{\prime \prime}(i)=i\right) \\
& \quad \times \mathbf{P}\left(M_{n-(k+i)}^{\prime \prime \prime}<0, L_{n-(k+i)}^{\prime \prime \prime} \geqslant x-y\right)
\end{aligned}
$$

Это соотношение, асимптотические представления (2), (3), свойства правильно меняющихся функций и лемма 7 приводят к оценкам

$$
\begin{aligned}
& \mathbf{P}\left(M_{n-(k+i)} \leqslant 0\right) \mathbf{P}\left(M_{k}<0\right) \mathbf{P}\left(S_{i} \geqslant \varepsilon^{(1-\delta) / \alpha} c_{n}, L_{i} \geqslant 0, \bar{\mu}(i)=i\right) \\
& \quad \leqslant C \mathbf{P}\left(M_{n-(k+i)} \leqslant 0\right) \mathbf{P}\left(M_{k}<0\right) \mathbf{P}^{2}\left(L_{i} \geqslant 0\right) i \mathbf{P}\left(X>\varepsilon^{(1-\delta) / \alpha} c_{n}\right) \\
& \quad \leqslant C_{1} \mathbf{P}\left(M_{n-(k+i)} \leqslant 0\right) \mathbf{P}\left(M_{k}<0\right) \mathbf{P}^{2}\left(L_{i} \geqslant 0\right) i\left(n \varepsilon^{1-\delta}\right)^{-1} \\
& \quad \leqslant C_{2} \mathbf{P}\left(M_{n-k} \leqslant 0\right) \mathbf{P}\left(M_{k}<0\right) \mathbf{P}^{2}\left(L_{i} \geqslant 0\right) i\left(n \varepsilon^{1-\delta}\right)^{-1} .
\end{aligned}
$$


Отсюда, с учетом (16) и свойств правильно меняющихся функций, заключаем, что при $n \rightarrow \infty$

$$
\begin{aligned}
R_{2-}(n, \varepsilon) & =\sum_{k=2 n \varepsilon}^{n(1-2 \varepsilon)} \sum_{i=1}^{n \varepsilon} \mathbf{P}(\tau(n)=k, \bar{\mu}(n)=k+i, \mathscr{A}(n, \varepsilon)) \\
& \leqslant \frac{C_{2}}{n \varepsilon^{1-\delta}} \sum_{k=2 n \varepsilon}^{n(1-2 \varepsilon)} \mathbf{P}\left(M_{n-k} \leqslant 0\right) \mathbf{P}\left(M_{k}<0\right) \sum_{i=1}^{n \varepsilon} i \mathbf{P}^{2}\left(L_{i} \geqslant 0\right) \\
& \leqslant C_{3} \frac{n^{2 \rho} \varepsilon^{2 \rho}}{n \varepsilon^{1-\delta} l_{1}^{2}(n \varepsilon)} \sum_{k=2 n \varepsilon}^{n(1-2 \varepsilon)} \mathbf{P}\left(M_{n-k} \leqslant 0\right) \mathbf{P}\left(M_{k}<0\right) \\
& \leqslant C_{3} \frac{n^{2 \rho} \varepsilon^{2 \rho}}{n \varepsilon^{1-\delta} l_{1}^{2}(n \varepsilon)} \mathbf{P}\left(M_{2 n \varepsilon} \leqslant 0\right) \sum_{k=2 n \varepsilon}^{n(1-2 \varepsilon)} \mathbf{P}\left(M_{k}<0\right) \\
& \leqslant C_{4} \frac{n^{2 \rho} \varepsilon^{2 \rho}}{n \varepsilon^{1-\delta} l_{1}^{2}(n \varepsilon)} \frac{1}{(2 n \varepsilon)^{\rho} l_{2}(2 n \varepsilon)} \frac{n^{1-\rho}}{l_{2}(n)} \\
& =\frac{C_{4}}{2^{\rho}} \frac{\varepsilon^{\rho-1+\delta}}{l_{1}^{2}(n \varepsilon) l_{2}(2 n \varepsilon) l_{2}(n)} \sim C_{5}\left(\frac{\sin \pi \rho}{\pi}\right)^{2} \varepsilon^{\rho-1+\delta} .
\end{aligned}
$$

Поскольку в рассматриваемом случае $0 \leqslant \bar{\mu}(n)-\tau(n) \leqslant \mu(n)-\tau(n)$, это доказывает равенство (25), если выбрать $\delta \in(1-\rho, 1)$, а затем устремить $\varepsilon$ к нулю.

Аналогичным образом можно оценить величину $R_{2+}(n, \varepsilon)$.

Лемма доказана.

Рассмотрим теперь четыре независимых случайных блуждания $\left\{S_{n}^{(i)}, n \geqslant 0\right\}, i=1,2,3,4$, с одним и тем же распределением приращений. Зафиксируем вектор $\mathbf{t}:=\left(t_{1}, t_{2}, t_{3}, t_{4}\right)$ с положительными компонентами и вектор $\mathbf{x}:=\left(x_{1}, x_{2}, x_{3}, x_{4}\right)$ с неотрицательными компонентами и введем события

$$
\mathscr{C}_{n, 1}(\mathbf{t}, \mathbf{x}):=\left\{M_{n t_{1}}^{(1)}<x_{1}, L_{n t_{2}}^{(2)} \geqslant-x_{2}, L_{n t_{3}}^{(3)} \geqslant-x_{3}, M_{n t_{4}}^{(4)}<x_{4}\right\}
$$

и

$$
\mathscr{C}_{n, 2}(\mathbf{t}):=\left\{\max \left\{-L_{n t_{1}}^{(1)}, M_{n t_{2}}^{(2)}, M_{n t_{3}}^{(3)},-L_{n t_{4}}^{(4)}\right\} \leqslant S_{n t_{2}}^{(2)}+S_{n t_{3}}^{(3)}\right\} .
$$

Пусть, далее, $L_{i}^{-}, L_{i}^{+}, i=1,2,3,4$, - независимые отрицательные и неотрицательные меандры строго устойчивых процессов Леви с параметром $\alpha$, а

$$
\mathfrak{l}_{i}:=\min _{0 \leqslant u \leqslant 1} L_{i}^{-}(u), \quad \mathfrak{m}_{i}:=\max _{0 \leqslant u \leqslant 1} L_{i}^{+}(u) .
$$

Лемма 6 влечет следующее утверждение.

Следствие 4. Если приращения случайных блужданий $\left\{S_{n}^{(i)}, n \geqslant\right.$ $0\}, i=1,2,3,4$, удовлетворяют условию $\mathrm{A} 2$, то для любого вектора $\mathbf{t}$ 
с положительньми компонентами и вектора $\mathbf{x}$ с неотрицательньми компонентами

$$
\lim _{n \rightarrow \infty} \mathbf{P}\left(\mathscr{C}_{n, 2}(\mathbf{t}) \mid \mathscr{C}_{n, 1}(\mathbf{t}, \mathbf{x})\right)=\mathbf{P}\left(\mathscr{C}_{2}(\mathbf{t})\right)
$$

где

$$
\mathscr{C}_{2}(\mathbf{t}):=\left\{\max \left\{-t_{1}^{1 / \alpha} \mathfrak{l}_{1}, t_{2}^{1 / \alpha} \mathfrak{m}_{2}, t_{3}^{1 / \alpha} \mathfrak{m}_{3},-t_{4}^{1 / \alpha} \mathfrak{l}_{4}\right\} \leqslant t_{2}^{1 / \alpha} L_{2}^{+}(1)+t_{3}^{1 / \alpha} L_{3}^{+}(1)\right\} .
$$

Для того, чтобы идти дальше, введем для $0 \leqslant k<l$ наборы отрезков случайной среды

$$
\mathbf{Q}_{+}(l, k):=\left(Q_{l+1}, \ldots, Q_{l+k}\right), \quad \mathbf{Q}_{-}(l, k):=\left(Q_{l}, \ldots, Q_{l-k}\right)
$$

и обозначим $\mathbf{Q}_{ \pm}^{(i)}(l, k), i=1,2,-$ независимые копии $\mathbf{Q}_{ \pm}(l, k)$.

Следующая лемма играет существенную роль в дальнейших рассуждениях.

Лемма 10. Если выполнень условия А2, то для фиксированного $k \geqslant 1$ распределение набора

$$
\left(\mathbf{Q}_{-}^{(1)}(\tau(n), k), \mathbf{Q}_{+}^{(1)}(\tau(n), k), \mathbf{Q}_{-}^{(2)}(\mu(n), k), \mathbf{Q}_{+}^{(2)}(\mu(n), k)\right)
$$

слабо сходится $к$ распределению $\mathscr{M}_{k}^{(1)} \otimes \mathscr{N}_{k}^{(1)} \otimes \mathscr{N}_{k}^{(2)} \otimes \mathscr{M}_{k}^{(2)}$, где $\mathscr{M}_{k}^{(i)}-$ распределение вектора $\mathbf{Q}^{(i)}(k):=\left(Q_{1}^{(i)}, \ldots, Q_{k}^{(i)}\right)$ относительно вероятностной меры $\mathbf{P}^{-}$, а $\mathscr{N}_{k}^{(i)}$ - распределение вектора $\mathbf{Q}^{(i)}(k)$ относительно вероятностной меры $\mathbf{P}^{+}$.

Д о к а з а т е л ь с т в о. В силу леммы 9 мы можем считать, что $|\tau(n)-\mu(n)| \geqslant 2 \varepsilon n$ для любого фиксированного $\varepsilon>0$, а по обобщенному закону арксинуса, что $\tau(n), \mu(n) \in[4 n \varepsilon, n(1-4 \varepsilon)]$ для достаточно малых значений $\varepsilon>0$. Дополнительные события имеют пренебрежимо малую вероятность, если сначала $n$ устремить к бесконечности, а затем $\varepsilon-\mathrm{k}$ нулю.

Обозначим

$$
\begin{aligned}
& \mathscr{A}_{1}=\mathscr{A}_{1}(\varepsilon, n):=\{(m, l): 4 n \varepsilon \leqslant m+2 \varepsilon n \leqslant l \leqslant n(1-4 \varepsilon)\} \\
& \mathscr{A}_{2}=\mathscr{A}_{2}(\varepsilon, n):=\{(m, l): 4 n \varepsilon \leqslant l+2 \varepsilon n \leqslant m \leqslant n(1-4 \varepsilon)\}
\end{aligned}
$$

и положим $\mathscr{A}=\mathscr{A}(\varepsilon, n):=\mathscr{A}_{1} \cup \mathscr{A}_{2}$.

В дальнейшем мы рассматриваем ситуацию $(m, l) \in \mathscr{A}_{1}$. Случай $(m, l) \in \mathscr{A}_{2}$ может быт исследован аналогичным способом путем перехода к отраженному случайному блужданию $\left\{-S_{k}, k \geqslant 0\right\}$.

Предполагая, что $(\tau(n), \mu(n))=(m, l) \in \mathscr{A}_{1}$, мы свяжем с первоначальным сопровождающим случайным блужданием $\left\{S_{j}, 0 \leqslant j \leqslant n\right\}$ 
следующие четыре случайных блуждания:

$$
\begin{aligned}
S_{j}^{\prime}:=S_{m}-S_{m-j}, & 0 \leqslant j \leqslant m, \\
S_{j}^{\prime \prime}:=S_{m+j}-S_{m}, & 0 \leqslant j \leqslant \frac{l-m}{2}, \\
S_{j}^{\prime \prime \prime}:=S_{l}-S_{l-j}, & 0 \leqslant j \leqslant \frac{l-m}{2}, \\
S_{j}^{\prime \prime \prime \prime}:=S_{l+j}-S_{l}, & 0 \leqslant j \leqslant n-l .
\end{aligned}
$$

Введем события

$$
\mathscr{D}_{1}:=\left\{M_{m}^{\prime}<0, L_{(l-m) / 2}^{\prime \prime} \geqslant 0, L_{(l-m) / 2}^{\prime \prime \prime} \geqslant 0, M_{n-l}^{\prime \prime \prime \prime}<0\right\}
$$

и

$$
\mathscr{D}_{2}:=\left\{\max \left\{-L_{m}^{\prime}, M_{(l-m) / 2}^{\prime \prime}, M_{(l-m) / 2}^{\prime \prime \prime},-L_{n-l}^{\prime \prime \prime \prime}\right\} \leqslant S_{(l-m) / 2}^{\prime \prime}+S_{(l-m) / 2}^{\prime \prime \prime}\right\} .
$$

Ясно, что если $(\tau(n), \mu(n))=(m, l)$, то $I\left\{\mathscr{D}_{1}\right\}=I\left\{\mathscr{D}_{2}\right\}=1$.

Пусть теперь $\psi_{i}: \mathscr{M}^{k} \rightarrow \mathbb{R}, i=1,2,3,4$, - ограниченные функции. Обозначим

$$
\begin{aligned}
\Psi_{k}(\tau(n), \mu(n)):= & \psi_{1}\left(\mathbf{Q}_{-}^{(1)}(\tau(n), k)\right) \psi_{2}\left(\mathbf{Q}_{+}^{(1)}(\tau(n), k)\right) \\
& \times \psi_{3}\left(\mathbf{Q}_{-}^{(2)}(\mu(n), k)\right) \psi_{4}\left(\mathbf{Q}_{+}^{(2)}(\mu(n), k)\right) .
\end{aligned}
$$

Имеем

$$
\begin{aligned}
\mathbf{E}\left[\Psi_{k}(\tau(n), \mu(n))\right] & =\sum_{m, l=0}^{n} \mathbf{E}\left[\Psi_{k}(\tau(n), \mu(n)) ; \tau(n)=m, \mu(n)=l\right] \\
& =\sum_{m, l=0}^{n} \mathbf{E}\left[\Psi_{k}(m, l) ; \mathscr{D}_{1} \cap \mathscr{D}_{2}\right],
\end{aligned}
$$

где

$$
\Psi_{k}(m, l):=\psi_{1}\left(\mathbf{Q}_{-}^{(1)}(m, k)\right) \psi_{2}\left(\mathbf{Q}_{+}^{(1)}(m, k)\right) \psi_{3}\left(\mathbf{Q}_{-}^{(2)}(l, k)\right) \psi_{4}\left(\mathbf{Q}_{+}^{(2)}(l, k)\right) .
$$

Введем теперь четыре независимых случайных блуждания $\left\{S_{j}^{(l)}, j=\right.$ $0,1, \ldots\}, l=1,2,3,4$, и для вектора $\mathbf{x}:=\left(x_{1}, x_{2}, x_{3}, x_{4}\right)$ с неотрицательными компонентами положим

$$
\begin{aligned}
\mathscr{D}_{1}^{*}= & \mathscr{D}_{1}^{*}\left(x_{1}, x_{2}, x_{3}, x_{4}\right)=\mathscr{D}_{1}^{*}(\mathbf{x}) \\
:= & \left\{M_{m-k}^{(1)}<x_{1}, L_{(l-m) / 2-k}^{(2)} \geqslant-x_{2}, L_{(l-m) / 2-k}^{(3)} \geqslant-x_{3}, M_{n-l-k}^{(4)}<x_{4}\right\}, \\
\mathscr{D}_{2}^{*}= & \mathscr{D}_{2}^{*}\left(x_{1}, x_{2}, x_{3}, x_{4}\right)=\mathscr{D}_{2}^{*}(\mathbf{x}) \\
:= & \left\{\operatorname { m a x } \left\{-L_{m-k}^{(1)}-x_{1}, M_{(l-m) / 2-k}^{(2)}+x_{2}, M_{(l-m) / 2-k}^{(3)}+x_{3},\right.\right. \\
& \left.\left.\quad-L_{n-l-k}^{(4)}-x_{4}\right\} \leqslant S_{(l-m) / 2-k}^{(2)}+S_{(l-m) / 2-k}^{(3)}+x_{2}+x_{3}\right\}
\end{aligned}
$$


И

$$
P\left(x_{1}, x_{2}, x_{3}, x_{4}\right)=P(\mathbf{x}):=\mathbf{P}\left(\mathscr{D}_{1}^{*} \cap \mathscr{D}_{2}^{*}\right) .
$$

Пусть, как и ранее, $\mathbf{Q}^{(i)}(k):=\left(Q_{1}^{(i)}, \ldots, Q_{k}^{(i)}\right)$, и пусть $\mathbf{Q}^{(i j)}(k), j=$ $1,2,-$ независимые копии наборов $\mathbf{Q}^{(i)}(k)$. Положим

$$
\Psi(k):=\psi_{1}\left(\mathbf{Q}^{(11)}(k)\right) \psi_{2}\left(\mathbf{Q}^{(12)}(k)\right) \psi_{3}\left(\mathbf{Q}^{(21)}(k)\right) \psi_{4}\left(\mathbf{Q}^{(22)}(k)\right) .
$$

Используя условные математические ожидания относительно $\sigma$-алгебр, порожденных первыми $k$ шагами всех четырех случайных блужданий (26)-(27), получаем

$$
\mathbf{E}\left[\Psi_{k}(m, l) ; \mathscr{D}_{1} \mathscr{D}_{2}\right]=\mathbf{E}\left[\Psi(k) I\left\{\mathscr{G}_{k}^{\prime}\right\} P\left(-S_{k}^{\prime}, S_{k}^{\prime \prime}, S_{k}^{\prime \prime \prime},-S_{k}^{\prime \prime \prime \prime}\right)\right],
$$

где

$$
\mathscr{G}_{k}^{\prime}:=I\left\{M_{k}^{\prime}<0, L_{k}^{\prime \prime} \geqslant 0, L_{k}^{\prime \prime \prime} \geqslant 0, M_{k}^{\prime \prime \prime}<0\right\} .
$$

Из наших условий следует, что для фиксированного $k$

$$
\lim _{x \rightarrow \infty} \mathbf{P}\left(\max \left\{\left|S_{k}^{\prime}\right|,\left|S_{k}^{\prime \prime}\right|,\left|S_{k}^{\prime \prime \prime}\right|,\left|S_{k}^{\prime \prime \prime \prime}\right|\right\}>x\right)=0 .
$$

С другой стороны, согласно лемме 6 , для любых $t>0$ и $y \in \mathbb{R}_{+}$

$$
\mathscr{L}\left(c_{n}^{-1} S_{n t}^{(i)} \mid L_{n t}^{(i)} \geqslant-y\right) \stackrel{w}{\rightarrow} \mathscr{L}\left(t^{1 / \alpha} L^{+}\right)
$$

при $n \rightarrow \infty$. Поскольку $l-m \geqslant 2 n \varepsilon$, то при условии $\left\{L_{n t}^{(i)} \geqslant-y\right\}$ и $l-m \rightarrow$ $\infty$ последовательность случайных величин $\min \left(S_{(l-m) / 2-k}^{(2)}, S_{(l-m) / 2-k}^{(3)}\right)$ стремится к бесконечности с вероятностью 1 . Это означает, что, пренебрегая событием, имеющим малую вероятность, мы можем заменить событие $D_{2}^{*}\left(S_{k}^{\prime}, S_{k}^{\prime \prime}, S_{k}^{\prime \prime \prime}, S_{k}^{\prime \prime \prime \prime}\right)$ событием

$$
\begin{aligned}
\mathscr{D}_{3}^{ \pm}= & \mathscr{D}_{3}^{ \pm}\left(\delta_{1}\right) \\
:=\left\{\max \left\{-L_{m-k}^{(1)}, M_{(l-m) / 2-k}^{(2)}, M_{(l-m) / 2-k}^{(3)},-L_{n-l-k}^{(4)}\right\}\right. & \left.\leqslant\left(1 \pm \delta_{1}\right)\left(S_{(l-m) / 2-k}^{(2)}+S_{(l-m) / 2-k}^{(3)}\right)\right\} \\
& \leqslant
\end{aligned}
$$

которое не зависит от $S_{k}^{\prime}, S_{k}^{\prime \prime}, S_{k}^{\prime \prime \prime}, S_{k}^{\prime \prime \prime \prime}$, и получить следующие оценки снизу и сверху для интересующего нас математического ожидания:

$$
\begin{aligned}
& \sum_{(m, l) \in \mathscr{A}_{1}} \mathbf{E}\left[\Psi(k) I\left\{\mathscr{G}_{k}^{\prime}\right\} \mathbf{P}\left(\mathscr{D}_{1}^{*}\left(S_{k}^{\prime}, S_{k}^{\prime \prime}, S_{k}^{\prime \prime \prime}, S_{k}^{\prime \prime \prime \prime}\right) \cap D_{3}^{-}\right)\right]-h(n, \varepsilon) \\
& \leqslant \mathbf{E}\left[\Psi_{k}(\tau(n), \mu(n)) ;(\tau(n), \mu(n)) \in \mathscr{A}_{1}\right] \\
& \leqslant \sum_{(m, l) \in \mathscr{A}_{1}} \mathbf{E}\left[\Psi(k) I\left\{\mathscr{G}_{k}^{\prime}\right\} \mathbf{P}\left(\mathscr{D}_{1}^{*}\left(S_{k}^{\prime}, S_{k}^{\prime \prime}, S_{k}^{\prime \prime \prime}, S_{k}^{\prime \prime \prime \prime}\right) \cap D_{3}^{+}\right)\right]+h(n, \varepsilon),
\end{aligned}
$$


где $h(n, \varepsilon) \rightarrow 0$, если сначала $n \rightarrow \infty$, а затем $\varepsilon \rightarrow 0$.

Положим

$$
P^{ \pm}(\mathbf{x})=P^{ \pm}\left(\mathbf{x}, \delta_{1}\right):=\mathbf{P}\left(\mathscr{D}_{1}^{*}(\mathbf{x}) \cap \mathscr{D}_{3}^{ \pm}\right) .
$$

Для завершения доказательства леммы 10, нам понадобится следующее утверждение.

Предложение 1. Если выполнены условия А1-А3, то для $m=$ $n t_{1}, l=n\left(t_{1}+2 t_{2}\right)$ и $t_{4}:=1-\left(t_{1}+2 t_{2}\right)>0$ справедливы оченки

$$
P^{ \pm}\left(\mathbf{x}, \delta_{1}\right) \leqslant C \mathbf{P}\left(\mathscr{D}_{1}\right) U\left(-x_{1}\right) V\left(x_{2}\right) V\left(x_{3}\right) U\left(-x_{4}\right)
$$

$u$

$$
\lim _{\delta_{1} \rightarrow 0} \lim _{n \rightarrow \infty} \frac{P^{ \pm}\left(\mathbf{x}, \delta_{1}\right)}{\mathbf{P}\left(\mathscr{D}_{1}\right)}=\mathbf{P}\left(\mathscr{C}_{2}(\mathbf{t})\right) U\left(-x_{1}\right) V\left(x_{2}\right) V\left(x_{3}\right) U\left(-x_{4}\right) .
$$

Д о к а з а т е л ь с т в о. Для доказательства первого неравенства заметим, что

$$
P^{ \pm}(\mathbf{x})=\mathbf{P}\left(\mathscr{D}_{1}^{*}(\mathbf{x})\right) \mathbf{P}\left(\mathscr{D}_{3}^{ \pm} \mid \mathscr{D}_{1}^{*}(\mathbf{x})\right) \leqslant \mathbf{P}\left(\mathscr{D}_{1}^{*}(\mathbf{x})\right)
$$

и, в силу (16), найдется константа $C$ такая, что

$$
\mathbf{P}\left(\mathscr{D}_{1}^{*}(\mathbf{x})\right) \leqslant C U\left(-x_{1}\right) V\left(x_{2}\right) V\left(x_{3}\right) U\left(-x_{4}\right) \mathbf{P}\left(\mathscr{D}_{1}\right) .
$$

Второе неравенство следует из (15), следствия 4, равенства

$$
\frac{P^{ \pm}(\mathbf{x})}{\mathbf{P}\left(\mathscr{D}_{1}\right)}=\frac{\mathbf{P}\left(\mathscr{D}_{1}^{*}(\mathbf{x})\right)}{\mathbf{P}\left(\mathscr{D}_{1}\right)} \mathbf{P}\left(\mathscr{D}_{3}^{ \pm} \mid \mathscr{D}_{1}^{*}(\mathbf{x})\right)
$$

и непрерывности распределений меандров устойчивых процессов Леви с параметром $\alpha$.

Возвращаясь к доказательству леммы 10, имеем

$$
\begin{aligned}
& \sum_{(m, l) \in \mathscr{A}_{1}} \mathbf{E}\left[\Psi(k) I\left\{\mathscr{G}_{k}^{\prime}\right\} \mathbf{P}\left(\mathscr{D}_{1}^{*}\left(S_{k}^{\prime}, S_{k}^{\prime \prime}, S_{k}^{\prime \prime \prime}, S_{k}^{\prime \prime \prime \prime}\right) \cap D_{3}^{ \pm}\right)\right] \\
& \quad=\sum_{(m, l) \in \mathscr{A}_{1}} \mathbf{E}\left[\Psi(k) I\left\{\mathscr{G}_{k}^{\prime}\right\} \frac{\mathbf{P}\left(\mathscr{D}_{1}^{*}\left(S_{k}^{\prime}, S_{k}^{\prime \prime \prime}, S_{k}^{\prime \prime \prime}, S_{k}^{\prime \prime \prime \prime}\right) \cap D_{3}^{ \pm}\right)}{\mathbf{P}\left(\mathscr{D}_{1}\right)}\right] \mathbf{P}\left(\mathscr{D}_{1}\right) .
\end{aligned}
$$

Нетрудно проверить, используя определения (26)-(27) и лемму 2 , что если $m=n t_{1}, l=n\left(t_{1}+2 t_{2}\right)$, то

$$
\begin{aligned}
\mathbf{P}\left(\mathscr{D}_{1}\right) & =\mathbf{P}\left(M_{m}^{\prime}<0, L_{(l-m) / 2}^{\prime \prime} \geqslant 0, L_{(l-m) / 2}^{\prime \prime \prime} \geqslant 0, M_{n-l}^{\prime \prime \prime \prime}<0\right) \\
& =\mathbf{P}\left(M_{m}<0\right) \mathbf{P}\left(L_{(l-m) / 2} \geqslant 0\right) \mathbf{P}\left(L_{(l-m) / 2} \geqslant 0\right) \mathbf{P}\left(M_{n-l}<0\right) \\
& \sim \frac{1}{n^{2}} g\left(t_{1}, t_{2}\right)
\end{aligned}
$$


равномерно по $(m, l) \in \mathscr{A}_{1}$, где

$$
g\left(t_{1}, t_{2}\right):=\left(\frac{\sin \pi \rho}{\pi}\right)^{2} \frac{1}{t_{1}^{\rho}} \frac{1}{t_{2}^{1-\rho}} \frac{1}{t_{2}^{1-\rho}} \frac{1}{\left(1-t_{1}-2 t_{2}\right)^{\rho}} .
$$

Положим

$$
\mathscr{B}_{1}(\varepsilon):=\left\{\left(t_{1}, t_{2}\right): 4 \varepsilon \leqslant t_{1}+2 \varepsilon \leqslant t_{1}+2 t_{2} \leqslant 1-4 \varepsilon\right\} .
$$

При $n \rightarrow \infty$ имеем

$$
\begin{aligned}
\sum_{(m, l) \in \mathscr{A}_{1}} \mathbf{E}\left[\Psi(k) I\left\{\mathscr{G}_{k}^{\prime}\right\}\right. & \left.\frac{\mathbf{P}\left(\mathscr{D}_{1}^{*}\left(S_{k}^{\prime}, S_{k}^{\prime \prime}, S_{k}^{\prime \prime \prime}, S_{k}^{\prime \prime \prime \prime}\right) \cap D_{3}^{ \pm}\right)}{\mathbf{P}\left(\mathscr{D}_{1}\right)}\right] \mathbf{P}\left(\mathscr{D}_{1}\right) \\
\sim \iint_{\left(t_{1}, t_{2}\right) \in \mathscr{B}_{1}(\varepsilon)} & \mathbf{E}\left[\Psi(k) I\left\{\mathscr{G}_{k}^{\prime}\right\} \frac{\mathbf{P}\left(\mathscr{D}_{1}^{*}\left(S_{k}^{\prime}, S_{k}^{\prime \prime}, S_{k}^{\prime \prime \prime}, S_{k}^{\prime \prime \prime \prime}\right) \cap D_{3}^{ \pm}\right)}{\mathbf{P}\left(\mathscr{D}_{1}\right)}\right] \\
& \times g\left(t_{1}, t_{2}\right) d t_{1} d t_{2} .
\end{aligned}
$$

Используя теорему о мажорируемой сходимости и (29), получаем

$$
\begin{aligned}
\lim _{n \rightarrow \infty} \iint_{\left(t_{1}, t_{2}\right) \in \mathscr{B}_{1}(\varepsilon)} & \mathbf{E}\left[\Psi(k) I\left\{\mathscr{G}_{k}^{\prime}\right\} \frac{\mathbf{P}\left(\mathscr{D}_{1}^{*}\left(S_{k}^{\prime}, S_{k}^{\prime \prime}, S_{k}^{\prime \prime \prime}, S_{k}^{\prime \prime \prime \prime}\right) \cap D_{3}^{ \pm}\right)}{\mathbf{P}\left(\mathscr{D}_{1}\right)}\right] \\
\times & \times g\left(t_{1}, t_{2}\right) d t_{1} d t_{2} \\
=\iint_{\left(t_{1}, t_{2}\right) \in \mathscr{B}_{1}(\varepsilon)} & \mathbf{E}\left[\Psi(k) I\left\{\mathscr{G}_{k}^{\prime}\right\} \lim _{n \rightarrow \infty} \frac{\mathbf{P}\left(\mathscr{D}_{1}^{*}\left(S_{k}^{\prime}, S_{k}^{\prime \prime}, S_{k}^{\prime \prime \prime}, S_{k}^{\prime \prime \prime \prime}\right) \cap D_{3}^{ \pm}\right)}{\mathbf{P}\left(\mathscr{D}_{1}\right)}\right] \\
\times & \times g\left(t_{1}, t_{2}\right) d t_{1} d t_{2} \\
=\iint_{\left(t_{1}, t_{2}\right) \in \mathscr{B}_{1}(\varepsilon)} & \mathbf{E}\left[\Psi(k) I\left\{\mathscr{G}_{k}^{\prime}\right\} U\left(-S_{k}^{\prime}\right) V\left(S_{k}^{\prime \prime}\right) V\left(S_{k}^{\prime \prime \prime}\right) U\left(-S_{k}^{\prime \prime \prime \prime}\right)\right] \mathbf{P}\left(\mathscr{C}_{2}(\mathbf{t})\right) \\
\times & \times g\left(t_{1}, t_{2}\right) d t_{1} d t_{2} \\
=\mathbf{E}\left[\Psi(k) U\left(-S_{k}^{\prime}\right) V\left(S_{k}^{\prime \prime}\right) V\left(S_{k}^{\prime \prime \prime}\right) U\left(-S_{k}^{\prime \prime \prime \prime}\right) I\left\{\mathscr{G}_{k}^{\prime}\right\}\right] \int_{\left(t_{1}, t_{2}\right) \in \mathscr{B}_{1}(\varepsilon)}^{\times g\left(t_{1}, t_{2}\right) d t_{1} d t_{2} .} & \mathbf{P}\left(\mathscr{C}_{2}(\mathbf{t})\right)
\end{aligned}
$$

Если $(m, l) \in \mathscr{A}_{2}$, то можно использовать аналогичные рассуждения, применяя их к отраженному случайному блужданию $\left\{-S_{k}, k \geqslant 0\right\}$. Отсюда следует, что

$$
\begin{aligned}
\lim _{n \rightarrow \infty} \mathbf{E}\left[\Psi_{k}(\tau(n), \mu(n))\right]= & \mathbf{E}\left[\Psi(k) U\left(-S_{k}^{\prime}\right) V\left(S_{k}^{\prime \prime}\right) V\left(S_{k}^{\prime \prime \prime}\right) U\left(-S_{k}^{\prime \prime \prime \prime}\right) I\left\{\mathscr{G}_{k}\right\}\right] \\
= & \mathbf{E}^{-}\left[\psi_{1}\left(\mathbf{Q}^{(1)}(k)\right)\right] \mathbf{E}^{+}\left[\psi_{2}\left(\mathbf{Q}^{(1)}(k)\right)\right] \\
& \times \mathbf{E}^{+}\left[\psi_{3}\left(\mathbf{Q}^{(2)}(k)\right)\right] \mathbf{E}^{-}\left[\psi_{4}\left(\mathbf{Q}^{(2)}(k)\right)\right],
\end{aligned}
$$

что влечет утверждение леммы.

Д ок аз а т е л с с т в о т е о р е м ы 2. Вначале мы докажем соотношение (9). Пусть $\psi_{i}: \mathbb{R} \rightarrow \mathbb{R}, i=1,2$, 一 непрерывные функции на 
интервале $[0,1]$, без потери общности ограниченные единицей по абсолютной величине. Для $k \leqslant m$ положим

$$
\zeta^{(i)}[m, k]:=\frac{1-f_{m-k, m+k}^{(i)}(0)}{e^{S_{m}(i)-S_{m-k}(i)}}=\frac{1-f_{m-k, m}^{(i)}\left(f_{m, m+k}^{(i)}(0)\right)}{e^{S_{m}(i)-S_{m-k}(i)}},
$$

и пусть

$$
\mathscr{H}_{k}(\varepsilon):=\left\{\left|\zeta_{n}^{(1)}-\zeta^{(1)}[\tau(n), k]\right| \leqslant \varepsilon ;\left|\zeta_{n}^{(2)}-\zeta^{(2)}[\mu(n), k]\right| \leqslant \varepsilon\right\} .
$$

Тогда для любого фиксированного $k$

$$
\begin{aligned}
& \left|\mathbf{E}\left[\psi_{1}\left(\zeta_{n}^{(1)}\right) \psi_{2}\left(\zeta_{n}^{(2)}\right)\right]-\mathbf{E}\left[\psi_{1}\left(\zeta^{(1)}[\tau(n), k]\right) \psi_{2}\left(\zeta^{(2)}[\mu(n), k]\right)\right]\right| \\
& \leqslant \mathbf{P}\left(\left|\zeta_{n}^{(1)}-\zeta^{(1)}[\tau(n), k]\right|>\varepsilon\right)+\mathbf{P}\left(\left|\zeta_{n}^{(2)}-\zeta^{(2)}[\mu(n), k]\right|>\varepsilon\right) \\
& \quad+\mathbf{E}\left[\left|\psi_{1}\left(\zeta_{n}^{(1)}\right) \psi_{2}\left(\zeta_{n}^{(2)}\right)-\psi_{1}\left(\zeta^{(1)}[\tau(n), k]\right) \psi_{2}\left(\zeta^{(2)}[\mu(n), k]\right)\right| I\left\{\mathscr{H}_{k}(\varepsilon)\right\}\right] .
\end{aligned}
$$

В силу следствия 2 каждое из первых двух слагаемых в правой части этого выражения можно сделать сколь угодно малым, если устремить к бесконечности сначала $n$, а затем $k$. С другой стороны, ввиду равномерной непрерывности функций $\psi_{i}, i=1,2$, на интервале $[0,1]$, величина

$$
\mathbf{E}\left[\left|\psi_{1}\left(\zeta_{n}^{(1)}\right) \psi_{2}\left(\zeta_{n}^{(2)}\right)-\psi_{1}\left(\zeta^{(1)}[\tau(n), k]\right) \psi_{2}\left(\zeta^{(2)}[\mu(n), k]\right)\right| I\left\{\mathscr{H}_{k}(\varepsilon)\right\}\right]
$$

может быть сделана сколь угодно малой за счет выбора достаточно малого значения $\varepsilon>0$. Таким образом,

$$
\mathbf{E}\left[\psi_{1}\left(\zeta_{n}^{(1)}\right) \psi_{2}\left(\zeta_{n}^{(2)}\right)\right]=\mathbf{E}\left[\psi_{1}\left(\zeta^{(1)}[\tau(n), k]\right) \psi_{2}\left(\zeta^{(2)}[\mu(n), k]\right)\right]+\delta_{k, n},
$$

где $\lim _{k \rightarrow \infty} \lim \sup _{n \rightarrow \infty}\left|\delta_{k, n}\right|=0$.

Вспоминая определение $\widehat{\mathbf{E}}$ в лемме 1 и используя лемму 10 , получаем

$$
\lim _{n \rightarrow \infty} \mathbf{E}\left[\psi_{1}\left(\zeta^{(1)}[\tau(n), k]\right) \psi_{2}\left(\zeta^{(2)}[\mu(n), k]\right)\right]=\widehat{\mathbf{E}}\left[\psi_{1}\left(\zeta^{(1)}[k]\right)\right] \widehat{\mathbf{E}}\left[\psi_{2}\left(\zeta^{(2)}[k]\right)\right],
$$

где

$$
\zeta^{(i)}[k]:=\frac{1-f_{k, 0}^{(i,-)}\left(f_{0, k}^{(i,+)}(0)\right)}{e^{S_{k}(i,-)}},
$$

а итерации $f_{k, 0}^{(i,-)}(s)$ и $f_{0, k}^{(i,+)}(s)$ построены по независимым участкам $\left(Q_{1}^{(i)}, \ldots, Q_{k}^{(i)}\right)$ среды в соответствии с мерами $\mathbf{P}^{-}$и $\mathbf{P}^{+}$. Поскольку последовательность

$$
\frac{1-f_{k, 0}^{(i,-)}(s)}{e^{S_{k}(i,-)}}, \quad k=1,2, \ldots
$$


монотонно убывает по $k=1,2, \ldots$ и $s \in[0,1]$, то предел

$$
\zeta^{(i,-)}(s):=\lim _{k \rightarrow \infty} \frac{1-f_{k, 0}^{(i,-)}(s)}{e^{S_{k}(i,-)}}
$$

существует $\mathbf{P}^{-}$-п.н. С другой стороны, последовательность $f_{0, k}^{(i,+)}(0)$ монотонно возрастает по $k$ и, следовательно, предел

$$
q^{(i,+)}:=\lim _{k \rightarrow \infty} f_{0, k}^{(i,+)}(0)
$$

существует $\mathbf{P}^{+}$-п.н. В результате предел

$$
\lim _{k \rightarrow \infty} \zeta^{(i)}[k]=\zeta^{(i,-)}\left(q^{(i,+)}\right):=\zeta^{(i)}
$$

существует $\widehat{\mathbf{P}}$-п.н. Отсюда и из леммы 1 вытекает соотношение

$$
\lim _{k \rightarrow \infty} \widehat{\mathbf{E}}\left[\psi_{1}\left(\zeta^{(1)}[k]\right)\right] \widehat{\mathbf{E}}\left[\psi_{2}\left(\zeta^{(2)}[k]\right)\right]=\widehat{\mathbf{E}}\left[\psi_{1}\left(\zeta^{(1)}\right)\right] \widehat{\mathbf{E}}\left[\psi_{2}\left(\zeta^{(2)}\right)\right],
$$

что влечет

$$
\lim _{n \rightarrow \infty} \mathbf{E}\left[\psi_{1}\left(\zeta_{n}^{(1)}\right) \psi_{2}\left(\zeta_{n}^{(2)}\right)\right]=\widehat{\mathbf{E}}\left[\psi_{1}\left(\zeta^{(2)}\right)\right] \widehat{\mathbf{E}}\left[\psi_{2}\left(\zeta^{(2)}\right)\right] .
$$

Итак, независимость и сходимость (9), заявленные в теореме 2, доказаны. Тот факт, что предельные случайные величины в (9) положительны с вероятностью 1 установлен в теореме 1 работы [9].

Второе утверждение теоремы 2 мы докажем только для $t=1$. Соответствующие утверждения для $0<t<1$ могут быть выведены из теоремы 4, которая будет доказана в следующем разделе. Положим

$$
\begin{aligned}
\zeta^{(i)}[m, k](\lambda) & :=\frac{1-f_{m-k, m+k}^{(i)}\left(\exp \left\{-\lambda e^{S_{m+k}(i)-S_{m}(i)}\right\}\right)}{e^{S_{m}(i)-S_{m-k}(i)}} \\
& =\frac{1-f_{m-k, m}^{(i)}\left(f_{m, m+k}^{(i)}\left(\exp \left\{-\lambda e^{S_{m+k}(i)-S_{m}(i)}\right\}\right)\right)}{e^{S_{m}(i)-S_{m-k}(i)}}
\end{aligned}
$$

и пусть $\zeta^{(i)}[m, k]=\zeta^{(i)}[m, k](\infty)$. Заметим сначала, что согласно лемме 10

$$
\begin{aligned}
& \left(\zeta^{(1)}[\tau(n), k]\left(\lambda_{1}\right), \zeta^{(1)}[\tau(n), k], \zeta^{(2)}[\mu(n), k]\left(\lambda_{2}\right), \zeta^{(2)}[\mu, k]\right) \\
& \quad \stackrel{d}{\rightarrow}\left(\zeta^{(1)}[k]\left(\lambda_{1}\right), \zeta^{(1)}[k], \zeta^{(2)}[k]\left(\lambda_{2}\right), \zeta^{(2)}[k]\right)
\end{aligned}
$$

при $n \rightarrow \infty$, где

$$
\zeta^{(i)}[k]\left(\lambda_{i}\right):=\frac{1-f_{k, 0}^{(i,-)}\left(f_{0, k}^{(i,+)}\left(\exp \left\{-\lambda e^{-S_{k}(i,+)}\right\}\right)\right)}{e^{S_{k}(i,-)}}
$$


и векторы $\left(\zeta^{(i)}[k]\left(\lambda_{i}\right), \zeta^{(i)}[k]\right), i=1,2$, независимы.

Этот факт в сочетании с леммами 4 и 5 влечет существование независимых векторов $\left(\zeta^{(i)}\left(\lambda_{i}\right), \zeta^{(i)}\right), i=1,2$ таких, что

$$
\left(e^{-S_{\tau(n)}(i)}\left(1-f_{0, n}^{(i)}\left(\exp \left\{-\lambda_{i} e^{S_{\tau(n)}(i)-S_{n}(i)}\right\}\right), 1-f_{0, n}^{(i)}(0)\right)\right) \stackrel{d}{\rightarrow}\left(\zeta^{(i)}\left(\lambda_{i}\right), \zeta^{(i)}\right) .
$$

Используя это соотношение и равенство

$$
\Phi_{n}(\lambda):=\mathbf{E}_{\pi}\left[e^{-\lambda \widehat{Y}_{n}} \mid Z_{n}>0\right]=1-\frac{1-f_{0, n}\left(\exp \left\{-\lambda\left(1-f_{0, n}(0)\right) e^{-S_{n}}\right\}\right)}{1-f_{0, n}(0)}
$$

и повторяя рассуждения из теоремы 1 статьи [10] (см., в частности, формулы (3.1)-(3.6) указанной работы), нетрудно проверить, что $\left(\Phi_{n}^{(1)}\left(\lambda_{1}\right), \Phi_{n}^{(2)}\left(\lambda_{2}\right)\right) \Rightarrow\left(\Phi^{(1)}\left(\lambda_{1}\right), \Phi^{(2)}\left(\lambda_{2}\right)\right)$ при $n \rightarrow \infty$, где (случайные) преобразования Лапласа $\Phi^{(i)}\left(\lambda_{i}\right), i=1,2$, обладают свойствами, заявленными в теореме 2 для случая $t=1$.

3. Многомерные условные распределения. Для $b \geqslant 2$ и $\mathbf{t}=$ $\left(t_{0}, t_{1}, \ldots, t_{b}\right), \bar{\lambda}=\left(\lambda_{1}, \ldots, \lambda_{b}\right)$, таких, что $0=t_{0}<t_{1}<\cdots<t_{b}=1$ и $\lambda_{i} \geqslant 0, i=1, \ldots, b$, обозначим $N_{j}=n t_{j}$ и, вспоминая (5), положим

$$
\Phi_{n}(\mathbf{t}, \bar{\lambda}):=\mathbf{E}_{\pi}\left[\exp \left\{-\sum_{i=1}^{b} \lambda_{i} \widehat{Y}_{N_{i}}\right\} \mid Z_{n}>0\right]
$$

и

$$
\Psi_{n}(\mathbf{t}, \bar{\lambda}):=\mathbf{E}_{\pi}\left[\widehat{Y}_{n} \exp \left\{-\sum_{i=1}^{b} \lambda_{i} \widehat{Y}_{N_{i}}\right\} \mid Z_{n}>0\right] .
$$

Для натуральных $d \leqslant b^{(1)}$ и $e \leqslant b^{(2)}$ и наборов неотрицательных целых чисел $\mathbf{P}=\left(p_{0}, p_{1}, p_{2}, \ldots, p_{d}\right)$ and $\mathbf{r}=\left(r_{0}, r_{1}, r_{2}, \ldots, r_{e}\right)$ таких, что

$$
0=p_{0}<p_{1}<p_{2}<\cdots<p_{d}=b^{(1)}, \quad 0=r_{0}<r_{1}<r_{2}<\cdots<r_{e}=b^{(2)}
$$

введем события

$$
\begin{aligned}
& \mathscr{U}^{(1)}(\mathbf{t} ; \mathbf{P}, n):=\{ \tau\left(N_{1}\right)=\cdots=\tau\left(N_{p_{1}}\right)<\tau\left(N_{p_{1}+1}\right)=\cdots \\
&=\left.\tau\left(N_{p_{2}}\right)<\cdots=\tau\left(N_{p_{d-1}}\right)<\tau\left(N_{p_{d-1}+1}\right)=\cdots=\tau\left(N_{p_{d}}\right)\right\} \\
& \mathscr{U}^{(2)}(\mathbf{t} ; \mathbf{r}, n):=\left\{\mu\left(N_{1}\right)=\cdots=\mu\left(N_{r_{1}}\right)<\mu\left(N_{r_{1}+1}\right)=\cdots\right. \\
&\left.=\mu\left(N_{r_{2}}\right)<\cdots=\mu\left(N_{r_{e-1}}\right)<\mu\left(N_{r_{e-1}+1}\right)=\cdots=\mu\left(N_{r_{e}}\right)\right\}
\end{aligned}
$$

и положим

$$
\mathscr{U}(\mathbf{t} ; \mathbf{P}, \mathbf{r}, n):=\mathscr{U}^{(1)}(\mathbf{t} ; \mathbf{P}, n) \cap \mathscr{U}^{(2)}(\mathbf{t} ; \mathbf{r}, n) .
$$

Основной результат данного раздела выглядит следующим образом (напомним, что мы используем верхние индексы $i=1,2$ для соответствующих характеристик и величин, связанных с ветвящимися процессами $Z^{(1)}$ и $\left.Z^{(2)}\right)$. 
Теорема 4. Если выполнень условия $\mathrm{A} 1-\mathrm{A} 3$, mо при $n \rightarrow \infty$

$$
\left\{\left(\Phi_{n}^{(1)}\left(\mathbf{t}, \bar{\lambda}^{(1)}\right), \Phi_{n}^{(2)}\left(\mathbf{t}, \bar{\lambda}^{(2)}\right)\right) \mid \mathscr{U}(\mathbf{t} ; \mathbf{P}, \mathbf{r}, n)\right\} \Rightarrow\left(\Phi^{(1)}\left(\bar{\lambda}^{(1)}\right), \Phi^{(2)}\left(\bar{\lambda}^{(2)}\right)\right)
$$

где $\Phi^{(i)}\left(\bar{\lambda}^{(i)}\right), i=1,2,-($ случайнье $)$ преобразования Лапласа собственньцх случайньх векторов. Кроме того, случайные функиии $\Phi^{(i)}\left(\bar{\lambda}^{(i)}\right)$, $i=1,2$, независимьл.

Заметим, что предельные случайные величины в (32) не зависят от $\mathbf{t}$.

Для доказательства этой теоремы напомним сначала ряд результатов, заимствованных из [10], для ветвящихся процессов с одним типом частиц.

Обозначим $Z_{n}(k)$ количество частиц в момент $n$ в ветвящемся процессе с одним типом частиц, стартующем с одной частицы в момент $k \leqslant n$. Положим

$$
\begin{gathered}
\widehat{Y}_{n}(k):=\frac{Z_{n}(k)}{\mathbf{E}_{\pi}\left[Z_{n}(k) \mid Z_{n}(k)>0\right]}, \\
\Phi_{k, n, \pi}(\lambda)=\Phi_{k, n}(\lambda):=\mathbf{E}_{\pi}\left[e^{-\lambda \widehat{Y}_{n}(k)} \mid Z_{n}(k)>0\right], \quad \Phi_{n}(\lambda):=\Phi_{0, n}(\lambda),
\end{gathered}
$$

и пусть

$$
\Psi_{k, n}(\lambda):=\mathbf{E}_{\pi}\left[\widehat{Y}_{n}(k) e^{-\lambda \widehat{Y}_{n}(k)} \mid Z_{n}(k)>0\right], \quad \Psi_{n}(\lambda):=\Psi_{0, n}(\lambda) .
$$

Следующие два результата, описывающие свойства (случайных) преобразований Лапласа $\Psi_{n}(\lambda)$ и $\Phi_{k, n}(\lambda)$, были доказаны в лемме 7 и теореме 3 работы [10], соответственно.

Лемма 11. Если выполнены условия А2-A3, то $\Psi_{n}(\cdot) \Rightarrow-\Phi^{\prime}(\cdot)$, $n \rightarrow \infty$, где функиия $\Phi$ та же, что и в (8).

Лемма 12. Если выполнены условия А2-А3, то для любых $\Lambda>1$ $u \varepsilon>0$

$$
\lim _{k \rightarrow \infty} \lim \sup _{n \rightarrow \infty} \mathbf{P}\left(\sup _{1 \leqslant \lambda \leqslant \Lambda}\left|\Psi_{\tau(n)-k, \tau(n)+k}(\lambda)-\Psi_{0, n}(\lambda)\right|>\varepsilon\right)=0 .
$$

Следующее утверждение легко вывести из следствия 7 статьи [10] и леммы 5 данной работы.

Лемма 13. Если выполнены условия А2-А3, то для любьх $\Lambda>1$ $u \varepsilon>0$

$$
\lim _{k \rightarrow \infty} \lim \sup _{n \rightarrow \infty} \mathbf{P}\left(\sup _{1 \leqslant \lambda \leqslant \Lambda}\left|\Phi_{\tau(n)-k, \tau(n)+k}(\lambda)-\Phi_{0, n}(\lambda)\right|>\varepsilon\right)=0 .
$$

Объединяя следствия 8 и 9 работы [10], легко убедиться в справедливости такого утверждения: 
Лемма 14. Если выполнены условия А2-А3, то для любых $\Lambda>1$ $u \varepsilon>0$

$$
\lim _{n \rightarrow \infty} \mathbf{P}\left(\sup _{\overline{1} \leqslant \bar{\lambda} \leqslant \bar{\Lambda}}\left|\Phi_{n}\left(\sum_{j=1}^{d} \lambda_{i}\right)-\Phi_{n}(\mathbf{t}, \bar{\lambda})\right|>\varepsilon ; \tau(n)<N_{1}\right)=0
$$

$u$

$$
\lim _{n \rightarrow \infty} \mathbf{P}\left(\sup _{\overline{1} \leqslant \bar{\lambda} \leqslant \bar{\Lambda}}\left|\Psi_{n}\left(\sum_{j=1}^{d} \lambda_{i}\right)-\Psi_{n}(\mathbf{t}, \bar{\lambda})\right|>\varepsilon ; \tau(n)<N_{1}\right)=0 .
$$

Упомянем, наконец, еще один результат, содержащийся в лемме 25 из [10]. Положим

$$
\mathscr{U}(\mathbf{t} ; n)=:\left\{0<\tau\left(N_{1}\right)<\tau\left(N_{2}\right)<\cdots<\tau\left(N_{d}\right)=\tau(n)\right\} .
$$

Лемма 15. Если выполнены условия А2-А3, то для любых $\Lambda>1$ $u \varepsilon>0$

$$
\lim _{n \rightarrow \infty} \mathbf{P}\left(\sup _{1 \leqslant \min _{i} \lambda_{i} \leqslant \Lambda}\left|\Phi_{n}(\bar{t}, \bar{\lambda})-\Phi_{N_{d-1}, N_{d}}\left(\lambda_{d}\right) \prod_{j=1}^{d-1} \Psi_{N_{j-1}, N_{j}}\left(\lambda_{j}\right)\right|>\varepsilon \mid \mathscr{U}(\mathbf{t} ; n)\right)=0 .
$$

Обозначим

$$
\lambda_{\mathbf{j}}(\mathbf{p}]:=\sum_{\mathbf{k}=\mathbf{p}_{\mathbf{j}-1}+\mathbf{1}}^{\mathbf{p}_{\mathbf{j}}} \lambda_{\mathbf{k}} .
$$

Из лемм 14 и 15 вытекает, что для любых $\Lambda>1$ и $\varepsilon>0$ при $n \rightarrow \infty$

$$
\begin{aligned}
& \mathbf{P}\left(\sup _{1 \leqslant \min _{i} \lambda_{i} \leqslant \Lambda}\left|\Phi_{n}(\mathbf{t}, \bar{\lambda})-\Phi_{N_{p_{d-1}}, N_{p_{d}}}\left(\lambda_{\mathbf{d}}(\mathbf{p}]\right) \prod_{\mathbf{j}=\mathbf{1}}^{\mathbf{d}-\mathbf{1}} \mathbf{\Psi}_{\mathbf{N}_{\mathbf{p}_{\mathbf{j}-1}}, \mathbf{N}_{\mathbf{p}_{\mathbf{j}}}}\left(\lambda_{\mathbf{j}}(\mathbf{p}]\right)\right|\right. \\
& >\varepsilon \mid \mathscr{U}(\mathbf{t} ; \mathbf{P}, n)) \rightarrow 0 .
\end{aligned}
$$

Обозначим $\mu(k, l)$ последний момент, когда случайное блуждание $\left\{S_{j}, k \leqslant j \leqslant l\right\}$ достигает своего максимального значения на интервале $[k, l]$, и пусть $\tau(k, l)$ - первый момент, когда случайное блуждание $\left\{S_{j}, k \leqslant j \leqslant l\right\}$ достигает своего минимального значения на этом интервале. Положим

$$
\begin{aligned}
\Xi^{(1)}(k, \mathbf{N}, \bar{\lambda}):= & \Phi_{\tau\left(N_{p_{d-1}}, N_{p_{d}}\right)-k, \tau\left(N_{p_{d-1}}, N_{p_{d}}\right)+k}^{(1)}\left(\lambda_{\mathbf{d}}(\mathbf{p}]\right) \\
& \times \prod_{j=1}^{d-1} \Psi_{\tau\left(N_{p_{j-1}}, N_{p_{j}}\right)-k, \tau\left(N_{p_{j-1}}, N_{p_{j}}\right)+k}^{(1)}\left(\lambda_{\mathbf{j}}(\mathbf{p}]\right)
\end{aligned}
$$

и

$$
\begin{aligned}
\Xi^{(2)}(k, \mathbf{N}, \bar{\lambda}):= & \Phi_{\mu\left(N_{r_{e-1}}, N_{r_{e}}\right)-k, \mu\left(N_{r_{e-1}}, N_{r_{e}}\right)+k}^{(2)}\left(\lambda_{\mathbf{e}}(\mathbf{r}]\right) \\
& \times \prod_{l=1}^{e-1} \Psi_{\mu\left(N_{r_{l-1}}, N_{r l}\right)-k, \mu\left(N_{r_{l-1}}, N_{r_{l}}\right)+k}^{(2)}\left(\lambda_{\mathbf{j}}(\mathbf{r}]\right) .
\end{aligned}
$$


По лемме 10 функции, входящие в $\Xi^{(i)}(k, \mathbf{N}, \bar{\lambda})$ в качестве множителей, асимптотически независимы, если $\left[N_{p_{j-1}}, N_{p_{j}}\right] \cap\left[N_{r_{l-1}}, N_{r_{l}}\right] \neq \oslash$ для некоторых $j$ и $l$, и, очевидно, независимы, если интервалы $\left[N_{p_{j-1}}, N_{p_{j}}\right]$ и $\left[N_{r_{l-1}}, N_{r_{l}}\right]$ не пересекаются. Более того, пределы указанных множителей при $n \rightarrow \infty$ не зависят от $\mathbf{t}$.

Отсюда следует, что при $n \rightarrow \infty$

$$
\begin{gathered}
\left\{\left(\Xi^{(1)}\left(k, \mathbf{N}, \bar{\lambda}^{(1)}\right), \Xi^{(2)}\left(k, \mathbf{N}, \bar{\lambda}^{(2)}\right)\right) \mid \mathscr{U}(\mathbf{t} ; \mathbf{p}, \mathbf{r}, n)\right\} \\
\Rightarrow\left(\Xi^{(1)}\left(k, \bar{\lambda}^{(1)}\right), \Xi^{(2)}\left(k, \bar{\lambda}^{(2)}\right)\right),
\end{gathered}
$$

где $\Xi^{(1)}\left(k, \bar{\lambda}^{(1)}\right), \Xi^{(2)}\left(k, \bar{\lambda}^{(2)}\right)$ - независимые случайные величины.

$\mathrm{C}$ другой стороны, в соответствии с леммой 12 и равенством (34)

$\lim _{k \rightarrow \infty} \lim \sup _{n \rightarrow \infty} \mathbf{P}\left(\sup _{1 \leqslant \sin _{j} \lambda_{j} \leqslant \Lambda}\left|\Phi_{n}^{(i)}(\mathbf{t}, \bar{\lambda})-\Xi^{(i)}(k, \mathbf{N}, \bar{\lambda})\right|>\varepsilon \mid \mathscr{U}^{(i)}(\mathbf{t} ; \mathbf{r}, n)\right)=0$.

При помощи этих двух соотношений, повторяя почти дословно доказательство теоремы 2 из [10], нетрудно убедиться в справедливости сходимости (32) и показать, что

$$
\begin{aligned}
& \Phi^{(1)}(\bar{\lambda})=(-1)^{d-1} \Phi_{d}^{(1)}\left(\lambda_{\mathbf{d}}(\mathbf{p}]\right) \prod_{\mathbf{j}=\mathbf{1}}^{\mathbf{d}-1} \Phi_{\mathbf{j}}^{(\mathbf{1}) \prime}\left(\lambda_{\mathbf{j}}(\mathbf{p}]\right), \\
& \Phi^{(2)}(\bar{\lambda})=(-1)^{e-1} \Phi_{e}^{(1)}\left(\lambda_{\mathbf{e}}(\mathbf{r}]\right) \prod_{\mathbf{l}=\mathbf{1}}^{\mathbf{e}-\mathbf{1}} \mathbf{\Phi}_{\mathbf{l}}^{(\mathbf{2}) \prime}\left(\lambda_{\mathbf{l}}(\mathbf{r}]\right),
\end{aligned}
$$

где случайные функции $\Phi_{j}^{(1)}, j=1, \ldots, d$, и $\Phi_{j}^{(2)}, j=1, \ldots, e$, взаимно независимы. Более того, они одинаково распределены внутри каждой группы, а $\Phi_{j}^{(1) \prime}, j=1, \ldots, d-1$ и $\Phi_{j}^{(2) \prime}, j=1, \ldots$, е являются производными независимых функций.

Тот факт, что соответствующие распределения - собственные, установлен в [10]. Теорема 4 доказана.

4. Совместные распределения в случайные моменты. В этом разделе мы докажем теорему 3 , описывающую распределение пары $\left(Z_{\tau(n t)}^{(1)}, Z_{\tau(n t)}^{(2)}\right)$ при условии, что оба типа не выродились к моменту $n$.

Из определения (11) следует, что

$$
\varphi_{\tau(n t), n}^{(1)}(s)=\frac{1-f_{0, \tau(n t)}^{(1)}\left(s f_{\tau(n t), n}^{(1)}(0)\right)}{1-f_{0, n}^{(1)}(0)}-\frac{1-f_{0, \tau(n t)}^{(1)}(s)}{1-f_{0, n}^{(1)}(0)} .
$$

Вначале мы рассмотрим случай $\tau(n)=\tau(n t), \mu(n)<\tau(n)$. Будем предполагать без ограничения общности, что $\tau(n)$ и $\mu(n)$ принадлежат интервалу $[4 \varepsilon n, n(1-4 \varepsilon)]$ для достаточно малого значения $\varepsilon$. Более того, согласно лемме 9 мы можем предполагать, что $\mu(n)<\tau(n)-2 \varepsilon n$. 
Если $\tau(n)=\tau(n t)$, то, в соответствии со следствием 2, мы можем заменить $\varphi_{\tau(n t), n}^{(1)}(s)$ величиной

$$
\varphi_{\tau(n t), n}^{(1)}(k ; s):=\frac{1-f_{\tau(n)-k, \tau(n)}^{(1)}\left(s f_{\tau(n), \tau(n)+k}^{(1)}(0)\right)}{1-f_{\tau(n)-k, \tau(n)+k}^{(1)}(0)}-\frac{1-f_{\tau(n)-k, \tau(n)}^{(1)}(s)}{1-f_{\tau(n)-k, \tau(n)+k}^{(1)}(0)},
$$

если $k$ достаточно велико. Заметим, что в силу следствий 2,3 и соотношений (30) и (31)

$$
\mathscr{L}\left(\varphi_{\tau(n t), n}^{(1)}(k ; s)\right) \stackrel{w}{\rightarrow} \mathscr{L}\left(\frac{\zeta^{(1,-)}\left(s q^{(1,+)}\right)-\zeta^{(1,-)}(s)}{\zeta^{(1,-)}\left(q^{(1,+)}\right)}\right):=\mathscr{L}\left(\varphi^{(1)}(s)\right)
$$

если вначале $n$, а затем $k$ устремить к бесконечности.

Для анализа второй допредельной компоненты в (12) заметим, что в силу неравенства

$$
\begin{gathered}
\left|\mathbf{E}_{\pi}\left[e^{-\lambda \widehat{Y}_{\tau(n t)}^{(2)}} \mid Z_{n}^{(2)}>0\right]-\mathbf{E}_{\pi}\left[e^{-\lambda \widehat{Y}_{n}^{(2)}} \mid Z_{n}^{(2)}>0\right]\right| \\
\leqslant \lambda \mathbf{E}_{\pi}\left[\left|\widehat{Y}_{\tau(n t)}^{(2)}-\widehat{Y}_{n}^{(2)}\right| \mid Z_{n}^{(2)}>0\right]
\end{gathered}
$$

оценки $\mu(n)<\tau(n)-2 \varepsilon n$ и леммы 19 в [10] мы можем заменить случайную величину $\widetilde{\Phi}_{\tau(n), n}^{(2)}(\lambda)$ на $\Phi_{0, n}^{(2)}(\lambda)$. В свою очередь, ввиду леммы 13 , $\Phi_{0, n}^{(2)}(\lambda)$ можно заменить величиной

$$
\Phi_{\mu(n)-k, \mu(n)-k}^{(2)}(\lambda)=\mathbf{E}_{\pi}\left[e^{-\lambda \widehat{Y}_{\mu(n)+k}^{(2)}} \mid Z_{\mu(n)-k}^{(2)}>0\right]
$$

(напомним, что точки минимума случайного блуждания $\left\{S_{k}(2), 0 \leqslant k \leqslant\right.$ $n\}$ это точки максимума случайного блуждания $\left.\left\{S_{k}(1), 0 \leqslant k \leqslant n\right\}\right)$.

Поскольку теперь интервалы $[\mu(n)-k, \mu(n)+k]$ и $[\tau(n)-k, \tau(n)+k]$ не пересекаются, мы можем воспользоваться леммой 10 и, устремив $k$ к бесконечности, заключить, что

$$
\begin{gathered}
\left(\varphi_{\tau(n), n}^{(1)}(k ; s), \Phi_{\mu(n)-k, \mu(n)-k}^{(2)}(\lambda)\right) I\{\mu(n)<\tau(n t)=\tau(n)\} \\
\Rightarrow\left(\varphi^{(1)}(s), \Phi^{(2)}(\lambda)\right) I\left\{\mu_{1}<\tau_{1}=\tau_{t}\right\}
\end{gathered}
$$

Это дает первое слагаемое в правой части (12).

Оставшиеся случаи могут быть рассмотрены аналогичным образом. При анализе первой компоненты допредельного вектора при условии $\tau(n t)<\tau(n)$ необходимо использовать соотношение

$$
\begin{aligned}
\varphi_{\tau(n t), n}^{(1)}(k ; s) & =\frac{f_{\tau(n t)-k, \tau(n t)}^{(1)}(s)-f_{\tau(n)-k, \tau(n t)}^{(1)}\left(s f_{\tau(n t), \tau(n t)+k}^{(1)}(0)\right)}{1-f_{\tau(n t)-k, \tau(n t)+k}^{(1)}(0)} \\
& \Rightarrow-s\left(\zeta^{(1,-)}(s)\right)^{\prime},
\end{aligned}
$$


справедливого, если сначала $n$, а затем $k$ устремить к бесконечности. Этот факт был установлен в теореме 6 из [11] при помощи неравенств

$$
\varphi_{\tau(n t), n}^{(1)}(k ; s) \leqslant s \frac{\left(f_{\tau(n t)-k, \tau(n t)}^{(1)}\right)^{\prime}(s)}{e^{S_{\tau(n t)}}} \frac{e^{S_{\tau(n t)-k}}\left(1-f_{\tau(n t), \tau(n t)+k}^{(1)}(0)\right)}{1-f_{\tau(n t)-k, \tau(n t)+k}^{(1)}(0)}
$$

и

$$
\begin{aligned}
\varphi_{\tau(n t), n}^{(1)}(k ; s) \geqslant & s \frac{\left(f_{\tau(n t)-k, \tau(n t)}^{(1)}\right)^{\prime}\left(s f_{\tau(n t), \tau(n t)+k}^{(1)}(0)\right)}{e^{S_{\tau(n t)}}} \\
& \times \frac{e^{S_{\tau(n t)-k}}\left(1-f_{\tau(n t), \tau(n t)+k}^{(1)}(0)\right)}{1-f_{\tau(n t)-k, \tau(n t)+k}^{(1)}(0)}
\end{aligned}
$$

и соотношений $(20)$ и $(21)$.

Для анализа первой компоненты допредельного вектора в теореме 3 в случае $\mu(n)>\tau(n t)$ положим

$$
\sigma_{\tau(n t), n}^{(2)}:=\frac{e^{S_{\tau(n t)}}\left(1-f_{\tau(n t), n}^{(2)}(0)\right)}{1-f_{0, n}^{(2)}(0)}
$$

и заметим, что при $\mathbf{m}_{n}^{*}(k):=\mathbf{E}_{\pi}\left[Z_{n}(k) \mid Z_{n}(k)>0\right]$

$$
\begin{aligned}
& \widetilde{\Phi}_{\tau(n t), n}^{(2)}(\lambda) \\
& \quad=\frac{f_{0, \tau(n t)}^{(2)}\left(\exp \left\{-\lambda \mathbf{m}_{n}^{*}(\tau(n t))\right\}\right)-f_{0, \tau(n t)}^{(2)}\left(\exp \left\{-\lambda \mathbf{m}_{n}^{*}(\tau(n t))\right\} f_{\tau(n t), n}^{(2)}(0)\right)}{1-f_{0, n}^{(2)}(0)} \\
& \quad \leqslant \sigma_{\tau(n t), n}^{(2)} \mathbf{E}_{\pi}\left[\widehat{Y}_{\tau(n t)}^{(2)} e^{-\lambda \widehat{Y}_{\tau(n t)}^{(2)}} \mid Z_{\tau(n t)}^{(2)}>0\right]
\end{aligned}
$$

и

$$
\begin{aligned}
\widetilde{\Phi}_{\tau(n t), n}^{(2)}(\lambda) \geqslant & \sigma_{\tau(n t), n}^{(2)} \mathbf{E}_{\pi}\left[\widehat{Y}_{\tau(n t)}^{(2)} e^{-\lambda \widehat{Y}_{\tau(n t)}^{(2)}}\left(f_{\tau(n t), n}^{(2)}(0)\right)^{Z_{\tau(n t)}^{(2)}} \mid Z_{\tau(n t)}^{(2)}>0\right] \\
& -\sigma_{\tau(n t), n}^{(2)} \mathbf{E}_{\pi}\left[\widehat{Y}_{\tau(n t)}^{(2)} e^{-\lambda \widehat{Y}_{\tau(n t)}^{(2)}}\left[1-\left(f_{\tau(n t), n}^{(2)}(0)\right)^{Z_{\tau(n t)}^{(2)}}\right] \mid Z_{\tau(n t)}^{(2)}>0\right] .
\end{aligned}
$$

Если $\mu(n)>\tau(n t)$, то силу леммы 11 и соотношения $(20) \sigma_{\tau(n t), n}^{(2)} \stackrel{\mathbf{P}}{\rightarrow} 1$ при $n \rightarrow \infty$, где $\stackrel{\mathbf{P}}{\rightarrow}$ означает сходимость по вероятности относительно меры P. Далее, $f_{\tau(n t), n}^{(2)}(0) I\{\mu(n)>\tau(n t)\} \stackrel{\text { P }}{\rightarrow} 1$ при $n \rightarrow \infty$ и, следовательно, если $\mu(n)>\tau(n t)$, то с вероятностью близкой к 1

$$
\left(f_{\tau(n t), n}^{(2)}(0)\right)^{Z_{\tau(n t)}^{(2)}} \geqslant \exp \left\{-2 Z_{\tau(n t)}^{(2)}\left(1-f_{\tau(n t), n}^{(2)}(0)\right)\right\} \geqslant e^{-2 W_{n}}
$$

где $W_{n}:=Z_{\tau(n t)}^{(2)} e^{-S_{\mu(n)}+S_{\tau(n t)}}$. Ясно, что $\mathbf{E}_{\pi}\left[W_{n}\right]=e^{-S_{\mu(n)}} \stackrel{p}{\rightarrow} 0, n \rightarrow \infty$. Отсюда вытекает, что для любого $\varepsilon \in(0,1)$

$$
\mathbf{P}\left(e^{-2 W_{n}}<1-\varepsilon\right)=\mathbf{P}\left(2 W_{n}>-\ln (1-\varepsilon)\right) \leqslant \frac{2 \mathbf{E} W_{n}}{-\ln (1-\varepsilon)}=\frac{2 \mathbf{E}\left[e^{-S_{\mu(n)}}\right]}{-\ln (1-\varepsilon)} \rightarrow 0
$$


при $n \rightarrow \infty$, что влечет $e^{-2 W_{n}} \stackrel{p}{\rightarrow} 1$. В результате имеем

$$
\left(f_{\tau(n t), n}^{(2)}(0)\right)^{Z_{\tau(n t)}^{(2)} \stackrel{p}{\rightarrow} 1}
$$

на множестве $\mu(n)>\tau(n t)$. Таким образом, вместо $\widetilde{\Phi}_{n t}^{(2)}(\lambda)$ достаточно исследовать поведение случайной величины

$$
\Psi_{\tau(n t), n}^{(2)}(\lambda)=\mathbf{E}_{\pi}\left[\widehat{Y}_{\tau(n t)}^{(2)} e^{-\lambda \widehat{Y}_{\tau(n t)}^{(2)}} \mid Z_{\tau(n t)}^{(2)}>0\right]
$$

при условии $\mu(n)>\tau(n t)$.

Используя формулу полной вероятности и лемму 12 , несложно показать, что $\Psi_{\tau(n t), n}(\lambda)$ можно заменить случайной функций $\Psi_{\mu(n)-k, \mu(n)+k}^{(2)}(\lambda)$. Как мы уже видели, интервалы $[\mu(n)-k, \mu(n)+k]$ и $[\tau(n)-k, \tau(n)+k]$ с вероятностью близкой к 1 не пересекаются для всех достаточно больших значений $n$ и подходящим образом выбранных $k$. Таким образом, для завершения доказательства достаточно применить лемму 10 и вспомнить лемму 11.

\section{СПИСОК ЛИТЕРАТУРЫ}

1. Afanasyev V.I., Geiger G., Kersting G., Vatutin V.A. Criticality for branching processes in random environment. - Ann. Probab., 2005, v. 33, № 2, p. 645-673.

2. Athreya K. B., Karlin S. On branching processes with random environments. I. Extinction probabilities. - Ann. Math. Statist., 1971, v. 42, p. 1499-1520.

3. Бородин А.Н., Салминен П. Справочник по броуновскому движению. Факты и формулы. СПб.: Лань, 2000, 640 с.

4. Doney R.A., Jones E. M. Large deviation results for random walks conditioned to stay positive. - J. London Mathem. Society, submitted.

5. Феллер В. Введение в теорию вероятностей и ее приложения, т. II. М.: Мир, 1984, $738 \mathrm{c.}$

6. Geiger J., Kersting G. The survival probability of a critical branching process in random environment. - Теория вероятн. и ее примен., 2000, т. 45, в. 3, с. 607-615.

7. Smith W. L., Wilkinson W. On branching processes in random environment. - Ann. Math. Statist., 1969, v. 40, № 3, p. 814-827.

8. Спичер Ф. Принципы случайного блуждания. М.: Мир, 1969, 472 с.

9. Ватутин B. А., Дьяконова E. E. Ветвящиеся процессы Гальтона-Ватсона в случайной среде, I: предельные теоремы. - Теория вероятн. и ее применен., 2003, т. 48 , в. 2, с. $274-300$.

10. Ватутин B. А., Дьяконова E. E. Ветвящиеся процессы Гальтона-Ватсона в случайной среде, II: конечномерные распределения. - Теория вероятн. и ее применен., 2004, т. 49, в. 2, с. 231-268.

11. Ватутин B.А., Дьяконова E. Е. Ветвящиеся процессы в случайной среде и бутылочные горлышки в эволюции популяций. - Теория вероятн. и ее применен., 2006 , т. 51 , в. 1 , с. $22-46$. 